\title{
SUPPLEMENT TO "A NONSTATIONARY NONPARAMETRIC BAYESIAN APPROACH TO DYNAMICALLY MODELING EFFECTIVE CONNECTIVITY IN FUNCTIONAL MAGNETIC RESONANCE IMAGING EXPERIMENTS"
}

\author{
By SOURABH BHATTACHARYA*,‡ AND RANJAN MAITRA ${ }^{\dagger, \S}$ \\ Indian Statistical Institute ${ }^{\ddagger}$ and Iowa State University ${ }^{\S}$
}

\section{S-1. Additional Details on Methodology.}

S-1.1. Full conditionals of $\alpha_{i}, \beta_{i}(t), \sigma_{\epsilon}^{2}$ and $\sigma_{\omega}^{2}$. The full conditional of $\alpha_{i}$ is normally distributed with mean $\varsigma_{\left[\alpha_{i} \mid \cdot\right]}^{2}=\left(\sigma_{\epsilon}^{-2} \sum_{t=1}^{T}\left\{y_{i}(t)-x(t) \beta_{i}(t)\right\}+\mu_{i} \sigma_{\alpha}^{-2}\right)$ and variance $\varsigma_{\left[\alpha_{i} \mid \cdots\right]}^{2}=\left(T \sigma_{\epsilon}^{-2}+\sigma_{\alpha}^{-2}\right)^{-1}$. The full conditionals of the $\beta_{i}(t) \mathrm{s}$ are also normal with mean $\nu_{\left[\beta_{i}(t) \mid \cdots\right]}$ and variance $\varsigma_{\left[\beta_{i}(t) \mid \cdots\right]}^{2}$, given by the following:

$$
\begin{aligned}
\nu_{\left[\beta_{i}(1) \mid \cdots\right]=\varsigma_{\left[\beta_{i}(1) \mid \cdots\right]}^{2}} & \sigma_{\epsilon}^{-2} x(1)\left(y_{i}(1)-\alpha_{i}\right) \\
& \left.+\sigma_{\omega}^{-2} x(1) \sum_{\ell=1}^{R} \gamma_{\ell i}(2)\left[\beta_{\ell}(2) x(1) \sum_{k=1, k \neq i}^{R} \gamma_{\ell k}(2) \beta_{k}(1)\right]+\sigma_{\beta}^{-2} \bar{\beta}\right)
\end{aligned}
$$

where $\varsigma_{\left[\beta_{i}(1) \mid \cdots\right]}^{-2}=\sigma_{\epsilon}^{-2} x^{2}(1)+\sigma_{\omega}^{-2} x^{2}(1) \sum_{r=1}^{R} \gamma_{r i}^{2}(2)+\sigma_{\beta}^{-2}$, and

$\nu_{\left[\beta_{i}(T) \mid \cdots\right]}=\varsigma_{\left[\beta_{i}(T) \mid \cdots\right]}^{2}\left[\sigma_{\epsilon}^{2} x(T)\left\{y_{i}(T)-\alpha_{i}\right\}+\sigma_{\omega}^{-2}\left\{x(T-1) \sum_{j=1}^{R} \gamma_{i j}(T) \beta_{j}(T-1)\right\}\right]$

with $\varsigma_{\left[\beta_{i}(T) \mid \cdots\right]}^{-2}=\sigma^{-2} x^{2}(T)_{\epsilon}+\sigma_{w}^{-2}$. For $t=2, \ldots, T-1$,

$$
\begin{aligned}
\nu_{\left[\beta_{i}(t) \mid \cdots\right]}= & \varsigma_{\left[\beta_{i}(t) \mid \cdots\right]}^{2}\left(\sigma_{\epsilon}^{-2} x(t)\left(y_{i}(t)-\alpha_{i}\right)+\sigma_{\omega}^{-2} x(t-1) \sum_{j=1}^{R} \gamma_{i j}(t) \beta_{j}(t-1)\right. \\
& \left.+\sigma_{\omega}^{-2} x(t) \sum_{\ell=1}^{R} \gamma_{\ell i}(t+1)\left\{\beta_{\ell}(t+1)-x(t) \sum_{k=1, k \neq i}^{R} \gamma_{\ell k}(t+1) \beta_{k}(t)\right\}\right)
\end{aligned}
$$

${ }^{*}$ Sourabh Bhattacharya is Assistant Professor in Bayesian and Interdisciplinary Research Unit, Indian Statistical Institute.

${ }^{\dagger}$ Ranjan Maitra is Associate Professor in the Department of Statistics and Statistical Laboratory, Iowa State University. His research was supported in part by the National Science Foundation CAREER Grant \# DMS-0437555 and by the National Institutes of Health (NIH) award \#DC-0006740. 
and

$$
\varsigma_{\left[\beta_{i}(t) \mid \cdots\right]}^{-2}=\sigma_{\epsilon}^{-2} x^{2}(t)+\sigma_{\omega}^{-2}\left[1+x^{2}(t) \sum_{\ell=1}^{R} \gamma_{\ell i}^{2}(t+1)\right]
$$

respectively. Finally, the full conditionals of $\sigma_{\epsilon}^{2}$ and $\sigma_{\omega}^{2}$ are $I G\left(a+\sum_{i=1}^{R} \sum_{t=1}^{T}\left(y_{i}(t)-\right.\right.$ $\left.\left.\alpha_{i}-x(t) \beta_{i}(t)\right)^{2}, b+R T\right)$ and $I G\left(a+\sum_{i=1}^{R} \sum_{t=1}^{T}\left(\beta_{i}(t)-x(t-1)\left\{\sum_{k=1}^{R} \gamma_{i k}(t) \beta_{k}(t-\right.\right.\right.$ 1) $\left.\})^{2}, b+R T\right)$, respectively.

S-1.2. Full Conditional Distributions of Configuration Indicators. Observe that, taking into account the coincidences among $\boldsymbol{\Gamma}_{i j}$, one can re-write (9) as

$$
\left[\boldsymbol{\Gamma}_{i j} \mid \cdots\right] \sim q_{0}^{(i j)} \boldsymbol{G}_{i j}^{(T)}+\sum_{(k, \ell)}^{*} n_{k \ell} q_{*}^{(k \ell)} \delta_{\Gamma_{k \ell}^{*}}
$$

In the above, $q_{*}^{(k \ell)}$ is exactly the same as $q^{(k \ell)}$ but with $\boldsymbol{\Gamma}_{k \ell}$ replaced with distinct values $\boldsymbol{\Gamma}_{k \ell}^{*}$, the latter being a distinct member of the set $\mathcal{D}=\left\{\boldsymbol{\Gamma}_{i^{\prime} j^{\prime}}:\left(i^{\prime}, j^{\prime}\right) \neq\right.$ $(i, j)\}$. Let $\mathcal{I}$ denote the set of indices of the form $\left(i^{\prime}, j^{\prime}\right)$ of the aforementioned distinct random variables in $\mathcal{D}$. Also, let $n_{k \ell}=\#\left\{\left(i^{\prime}, j^{\prime}\right) \neq(i, j): \boldsymbol{\Gamma}_{i^{\prime} j^{\prime}}=\boldsymbol{\Gamma}_{k \ell}^{*}\right\}$, and $\sum_{(k, \ell)}^{*}$ denotes summation over the set $\mathcal{I}$. Note that, $\sum_{(k, \ell)}^{*} n_{k \ell}=\#\{(k, \ell)$ : $(k, \ell) \neq(i, j)\}$.

We can take advantage of representation (1) to simulate only the distinct elements $\Gamma_{k \ell}^{*}$, given simulated values of the configuration indicators, defined, for any $(i, j) \in\left\{\left(i^{\prime}, j^{\prime}\right): i^{\prime}=1, \ldots, R, j^{\prime}=1, \ldots, R\right\}$, by $\boldsymbol{c}_{i j}=(k, \ell)$ if and only if $\boldsymbol{\Gamma}_{i j}=\boldsymbol{\Gamma}_{k \ell}^{*}$. So, $n_{k \ell}$ may be defined alternatively as $n_{k \ell}=\#\left\{\left(i^{\prime}, j^{\prime}\right) \neq(i, j)\right.$ : $\left.\boldsymbol{c}_{i^{\prime} j^{\prime}}=(k, \ell)\right\}$. Now, from (1) it follows that the full conditional distribution of $\boldsymbol{c}_{i j}$ is given by

$$
\begin{aligned}
{\left[\boldsymbol{c}_{i j}=(k, \ell) \mid \cdots\right] } & \propto n_{k \ell} q_{*}^{(k \ell)} \text { if }(k, \ell) \in \mathcal{I} \\
& \propto q_{0}^{(i j)} \quad \text { if }(k, \ell) \notin \mathcal{I}
\end{aligned}
$$

The full conditionals are available, however, given configuration indicators, which we describe in the next section.

S-1.3. Full Conditional Distributions of the Distinct Values given Configuration Vectors. Given the configuration set $\boldsymbol{C}=\left\{\boldsymbol{c}_{i j}: i=1, \ldots, R ; j=1, \ldots, R\right\}$ simulated according to (2) or (3), we can simulate only the distinct $\boldsymbol{\Gamma}_{i j}^{*}$ 's, rather than the entire set $\left\{\boldsymbol{\Gamma}_{i j}: i=1, \ldots, R ; j=1, \ldots, R\right\}$. However, in this set up the distinct $\boldsymbol{\Gamma}_{i j}^{*}$ have (multivariate normal) distributions from which a general form of the parameters is difficult to identify, due to the lack of symmetry in our set up. For $R=3$ this means that, for each of possible $2^{9}$ configurations, we need to separately derive the parameters of the relevant multivariate normal distributions. We 
illustrate five cases below.

\section{Case 1:}

Let $\boldsymbol{C}=\{(1,1),(1,2),(1,3),(2,1),(2,2),(2,3),(3,1),(3,2),(3,3)\}$, that is, all $\boldsymbol{\Gamma}_{i j}$ are distinct. Then, given this particular configuration, for all $(i, j) ; i=1,2,3 ; j=$ $1,2,3$, we have the following distribution of $\boldsymbol{\Gamma}_{i j}^{*}$ :

$$
\left[\boldsymbol{\Gamma}_{i j}^{*} \mid \boldsymbol{C}, \cdots\right] \sim \boldsymbol{G}_{i j}^{(T)}
$$

\section{Case 2:}

$\boldsymbol{C}=\{(1,1),(1,1),(1,3),(2,1),(2,2),(2,3),(3,1),(3,2),(3,3)\}$, that is, except $\boldsymbol{\Gamma}_{11}$ and $\boldsymbol{\Gamma}_{12}$, which are equal, all others are distinct. In this case, it can be shown that the full conditional distribution of $\Gamma_{11}^{*}$ is a $T$-variate normal, given by

(5)

$$
\left[\boldsymbol{\Gamma}_{11}^{*} \mid \boldsymbol{C}, \cdots\right] \sim N_{T}\left\{\left(\boldsymbol{\Sigma}^{-1}+\boldsymbol{A}_{11}^{*}\right)^{-1}\left(\bar{\gamma} \boldsymbol{\Sigma}^{-1} \boldsymbol{\mu}_{T}+\boldsymbol{B}_{11}^{*}\right),\left(\boldsymbol{\Sigma}^{-1}+\boldsymbol{A}_{11}^{*}\right)^{-1}\right\}
$$

where

$$
\boldsymbol{A}_{11}^{*}=\frac{1}{\sigma_{w}^{2}} \operatorname{diag}\left\{0, x^{2}(1)\left(\beta_{1}(1)+\beta_{2}(t)\right)^{2}, \ldots, x^{2}(T-1)\left(\beta_{1}(T-1)+\beta_{2}(T-1)\right)^{2}\right\}
$$

The first element of the vector $\boldsymbol{B}_{11}^{*}, \boldsymbol{B}_{11}^{*}(1)=0$, and, for $t=2, \ldots, T$ the $t$-th elements of $\boldsymbol{B}_{11}^{*}$ are given by the following.

$$
\begin{aligned}
\boldsymbol{B}_{11}^{*}(t) & =\frac{1}{\sigma_{w}^{2}}\left\{x(t-1) \beta_{1}(t)\left(\beta_{1}(t-1)+\beta_{2}(t-1)\right)\right. \\
& \left.-x^{2}(t-1) \gamma_{13}(t) \beta_{3}(t-1)\left(\beta_{1}(t-1)+\beta_{2}(t-1)\right)\right\}
\end{aligned}
$$

The other distinct elements will be distributed as $\boldsymbol{G}_{i j}^{(T)}$ conditional on the remaining $\boldsymbol{\Gamma}_{i j}^{*}$, and replacing both $\boldsymbol{\Gamma}_{11}$ and $\boldsymbol{\Gamma}_{12}$ with $\boldsymbol{\Gamma}_{11}^{*}$.

\section{Case 3:}

$\boldsymbol{C}=\{(1,1),(1,2),(1,3),(2,1),(1,1),(2,3),(3,1),(3,2),(3,3)\}$, that is, $\boldsymbol{\Gamma}_{11}=$ $\boldsymbol{\Gamma}_{22}=\boldsymbol{\Gamma}_{11}^{*}$ (say), and the others are distinct. Then $\boldsymbol{\Gamma}_{11}^{*}$ has the same distributional form as (5) but with $\boldsymbol{A}_{11}^{*}$ and $\boldsymbol{B}_{11}^{*}$ replaced with

$$
\boldsymbol{A}_{11}^{*}=\frac{1}{\sigma_{w}^{2}} \operatorname{diag}\left\{0, x^{2}(1)\left(\beta_{1}^{2}(1)+\beta_{2}^{2}(t)\right), \ldots, x^{2}(T-1)\left(\beta_{1}^{2}(T-1)+\beta_{2}^{2}(T-1)\right)\right\}
$$


The first element of the vector $\boldsymbol{B}_{11}^{*}, \boldsymbol{B}_{11}^{*}(1)=0$, and, for $t=2, \ldots, T$ the $t$-th elements of $\boldsymbol{B}_{11}^{*}$ are given by the following.

$$
\begin{aligned}
\boldsymbol{B}_{11}^{*}(t) & =\frac{1}{\sigma_{w}^{2}}\left\{x(t-1) \beta_{1}(t) \beta_{1}(t-1)-x^{2}(t-1) \gamma_{12}(t) \beta_{1}(t-1) \beta_{2}(t-1)\right. \\
& -x^{2}(t-1) \gamma_{13}(t) \beta_{1}(t-1) \beta_{3}(t-1)+x(t-1) \beta_{2}(t-1) \beta_{2}(t) \\
& \left.-x^{2}(t-1) \gamma_{21}(t) \beta_{1}(t-1) \beta_{2}(t-1)-x^{2}(t-1) \gamma_{23}(t) \beta_{2}(t-1) \beta_{3}(t-1)\right\}
\end{aligned}
$$

As in Case 2, the other distinct elements will be distributed as $\boldsymbol{G}_{i j}^{(T)}$ conditional on the remaining $\boldsymbol{\Gamma}_{i j}^{*}$, and replacing both $\boldsymbol{\Gamma}_{11}$ and $\boldsymbol{\Gamma}_{22}$ with $\boldsymbol{\Gamma}_{11}^{*}$.

\section{Case 4:}

$\boldsymbol{C}=\{(1,1),(1,2),(1,3),(2,1),(1,1),(2,3),(3,1),(3,2),(1,1)\}$, that is, $\boldsymbol{\Gamma}_{11}=$ $\boldsymbol{\Gamma}_{22}=\boldsymbol{\Gamma}_{33}=\boldsymbol{\Gamma}_{11}^{*}$ (say); all others are distinct in this configuration. In this case as well, $\boldsymbol{\Gamma}_{11}^{*}$ has the same distributional form as (5) but with $\boldsymbol{A}_{11}^{*}$ and $\boldsymbol{B}_{11}^{*}$ replaced with

$$
\begin{aligned}
\boldsymbol{A}_{11}^{*} & =\frac{1}{\sigma_{w}^{2}} \operatorname{diag}\left\{0, x^{2}(1)\left(\beta_{1}^{2}(1)+\beta_{2}^{2}(t)+\beta_{3}^{2}(t)\right)\right. \\
& \left.\ldots, x^{2}(T-1)\left(\beta_{1}^{2}(T-1)+\beta_{2}^{2}(T-1)+\beta_{3}^{2}(T-1)\right)\right\}
\end{aligned}
$$

The first element of the vector $\boldsymbol{B}_{11}^{*}, \boldsymbol{B}_{11}^{*}(1)=0$, and, for $t=2, \ldots, T$ the $t$-th elements of $\boldsymbol{B}_{11}^{*}$ are given by the following.

$$
\begin{aligned}
\boldsymbol{B}_{11}^{*}(t) & =\frac{1}{\sigma_{w}^{2}}\left\{x(t-1) \beta_{1}(t) \beta_{1}(t-1)-x^{2}(t-1) \gamma_{12}(t) \beta_{1}(t-1) \beta_{2}(t-1)\right. \\
& -x^{2}(t-1) \gamma_{13}(t) \beta_{1}(t-1) \beta_{3}(t-1)+x(t-1) \beta_{2}(t-1) \beta_{2}(t) \\
& -x^{2}(t-1) \gamma_{21}(t) \beta_{1}(t-1) \beta_{2}(t-1)-x^{2}(t-1) \gamma_{23}(t) \beta_{2}(t-1) \beta_{3}(t-1) \\
& +x(t-1) \beta_{3}(t) \beta_{3}(t-1)-x^{2}(t-1) \gamma_{32}(t) \beta_{2}(t-1) \beta_{3}(t-1) \\
(11) & \left.x^{2}(t-1) \gamma_{31}(t) \beta_{1}(t-1) \beta_{3}(t-1)\right\}
\end{aligned}
$$

All other distinct elements will be distributed as $\boldsymbol{G}_{i j}^{(T)}$ conditional on the remaining $\boldsymbol{\Gamma}_{i j}^{*}$, and substituting $\boldsymbol{\Gamma}_{11}=\boldsymbol{\Gamma}_{22}=\boldsymbol{\Gamma}_{33}=\boldsymbol{\Gamma}_{11}^{*}$.

\section{Case 5:}

$\boldsymbol{C}=\{(1,1),(1,1),(1,1),(1,1),(1,1),(1,1),(1,1),(1,1),(1,1)\}$, that is, there is only one distinct element. Then, the full conditional distribution of $\Gamma_{11}^{*}$ is the 
same as (5) with forms of $\boldsymbol{A}_{11}^{*}$ and $\boldsymbol{B}_{11}^{*}$ replaced with

$$
\begin{aligned}
\boldsymbol{A}_{11}^{*} & =\frac{1}{\sigma_{w}^{2}} \operatorname{diag}\left\{0,3 x^{2}(1)\left(\beta_{1}(1)+\beta_{2}(t)+\beta_{3}(t)\right)^{2},\right. \\
& \left.\ldots, 3 x^{2}(T-1)\left(\beta_{1}(T-1)+\beta_{2}(T-1)+\beta_{3}(T-1)\right)^{2}\right\}
\end{aligned}
$$

The first element of the vector $\boldsymbol{B}_{11}^{*}, \boldsymbol{B}_{11}^{*}(1)=0$, and, for $t=2, \ldots, T$ the $t$-th elements of $\boldsymbol{B}_{11}^{*}$ are given by the following.

$$
\boldsymbol{B}_{11}^{*}(t)=\frac{1}{\sigma_{w}^{2}} x(t-1)\left\{\beta_{1}(t-1)+\beta_{2}(t-1)+\beta_{3}(t-1)\right\}\left\{\beta_{1}(t)+\beta_{2}(t)+\beta_{3}(t)\right\}
$$

\section{S-1.4. Full conditional distributions of $\sigma_{\delta}^{2}$ and $\rho$ given the configuration vector}

C. Let us define

$$
\boldsymbol{Q}=\sum_{i, j}^{*} \sum_{t=2}^{T}\left(\gamma_{i j}^{*}(t)-\rho \gamma_{i j}^{*}(t-1)\right)
$$

where $\sum_{i, j}^{*}$ indicates summation over all distinct elements $\boldsymbol{\Gamma}_{i j}^{*}$. Let $d$ denote the number of distinct elements among $\left\{\boldsymbol{\Gamma}_{i j} ; i, j=1,2,3\right\}$. Then, if a priori $\sigma_{\delta}^{2} \sim$ $I G(a, b)$, the full conditional distribution of $\sigma_{\delta}^{2}$ is given by

$$
\left[\sigma_{\delta}^{2} \mid \boldsymbol{C}, \cdots\right] \sim I G(\boldsymbol{Q}+a, b+d(T-1))
$$

Given a uniform prior of $\rho$ on $(-1,1)$, the full conditional distribution of $\rho$, given the configuration vector $C$, is truncated normal, given by

$$
[\rho \mid \boldsymbol{C}, \cdots] \sim N\left(\frac{\sum_{i, j}^{*} \sum_{t=2}^{T} \gamma_{i j}^{*}(t) \gamma_{i j}^{*}(t-1)}{\sum_{i, j}^{*} \sum_{t=2}^{T}\left\{\gamma_{i j}^{*}(t)\right\}^{2}}, \frac{\sigma_{\delta}^{2}}{\sum_{i, j}^{*} \sum_{t=2}^{T}\left\{\gamma_{i j}^{*}(t)\right\}^{2}}\right) I(-1<\rho<1)
$$

S-1.5. Model averaging. Let $\boldsymbol{Y}=\left\{\boldsymbol{y}_{1}, \ldots, \boldsymbol{y}_{T}\right\}$, where $\boldsymbol{y}_{t}=\left(y_{1}(t), \ldots, y_{R}(t)\right)^{\prime}$ for $t=1, \ldots, T$. We denote by $\boldsymbol{B}$ the set of all $\beta$ 's and for $(i, j)=1, \ldots, R$, let $\boldsymbol{\Gamma}_{i j}$ denote the set $\left\{\gamma_{i j}(t) ; t=1, \ldots, T\right\}$. Assume that the other parameters and hyperparameters are known. (This assumption is not necessary but simplifies notation.) Then, the conditional distribution of $\boldsymbol{B}$, given the random measure $\boldsymbol{G}^{(T)}$ is given by

$$
\left[\boldsymbol{B} \mid \boldsymbol{G}^{(T)}\right]=\int\left[\boldsymbol{B} \mid \boldsymbol{\Gamma}_{i j}: i=1, \ldots, R, j=1, \ldots, R\right] \prod_{i, j} \boldsymbol{G}^{(T)}\left(d \boldsymbol{\Gamma}_{i j}\right)
$$


Then the distribution of the data $\boldsymbol{Y}$ conditional on $\boldsymbol{G}^{(T)}$ is given by

$$
\left[\boldsymbol{Y} \mid \boldsymbol{G}^{(T)}\right]=\int[\boldsymbol{Y} \mid \boldsymbol{B}]\left[\boldsymbol{B} \mid \boldsymbol{G}^{(T)}\right] d \boldsymbol{B}
$$

The conditional model $\left[\boldsymbol{Y} \mid \boldsymbol{G}^{(T)}\right]$ implies that data $\boldsymbol{Y}$ is associated with distribution $\boldsymbol{G}^{(T)}$. Finally, the marginal distribution of $\boldsymbol{Y}$ is

$$
[\boldsymbol{Y}]=\int\left[\boldsymbol{Y} \mid \boldsymbol{G}^{(T)}\right] d\left[\boldsymbol{G}^{(T)}\right]
$$

Thus, $\boldsymbol{Y}$ is a mixture of models of the form $\left[\boldsymbol{Y} \mid \boldsymbol{G}^{(T)}\right]$, the mixing being over all distributions $\boldsymbol{G}^{(T)}$ contained in the support of the DP prior of $\boldsymbol{G}^{(T)}$. Hence, the marginal $[\boldsymbol{Y}]$ is a weighted average with respect to $\boldsymbol{G}^{(T)}$ of all models of the form $\left[\boldsymbol{Y} \mid \boldsymbol{G}^{(T)}\right]$, the associated weight being $\left[\boldsymbol{G}^{(T)}\right]$. Each model $\left[\boldsymbol{Y} \mid \boldsymbol{G}^{(T)}\right]$ indicates that data $\boldsymbol{Y}$ is associated with that particular $\boldsymbol{G}^{(T)}$.

Similar issue holds in the case of leave-one-out cross-validation posteriors. Let $\boldsymbol{Y}_{-t}=\left\{\boldsymbol{y}_{1}, \ldots, \boldsymbol{y}_{t-1}, \boldsymbol{y}_{t+1}, \ldots, \boldsymbol{y}_{T}\right\}$. Here we have,

$$
\left[\boldsymbol{y}_{t} \mid \boldsymbol{G}^{(T)}, \boldsymbol{Y}_{-t}\right]=\int\left[\boldsymbol{y}_{t} \mid \boldsymbol{B}\right]\left[\boldsymbol{B} \mid \boldsymbol{G}^{(T)}, \boldsymbol{Y}_{-t}\right] d \boldsymbol{B},
$$

where

$$
\left[\boldsymbol{B} \mid \boldsymbol{G}^{(T)}, \boldsymbol{Y}_{-t}\right]=\int\left[\boldsymbol{B} \mid \boldsymbol{\Gamma}, \boldsymbol{Y}_{-t}\right] d\left[\boldsymbol{\Gamma} \mid \boldsymbol{G}^{(T)}, \boldsymbol{Y}_{-t}\right],
$$

where $\boldsymbol{\Gamma}=\left\{\boldsymbol{\Gamma}_{i j} ;(i, j)=1, \ldots, R\right\}$. Here

$$
\left[\boldsymbol{\Gamma} \mid \boldsymbol{G}^{(T)}, \boldsymbol{Y}_{-t}\right]=\frac{\left[\boldsymbol{Y}_{-t} \mid \boldsymbol{G}^{(T)}, \boldsymbol{\Gamma}\right] \prod_{i, j} \boldsymbol{G}^{(T)}\left(\boldsymbol{\Gamma}_{i j}\right)}{\left[\boldsymbol{Y}_{-t} \mid \boldsymbol{G}^{(T)}\right]}
$$

with

$$
\left[\boldsymbol{Y}_{-t} \mid \boldsymbol{G}^{(T)}, \boldsymbol{\Gamma}\right]=\int\left[\boldsymbol{Y}_{-t} \mid \boldsymbol{B}\right][\boldsymbol{B} \mid \boldsymbol{\Gamma}] d \boldsymbol{B}=\left[\boldsymbol{Y}_{-t} \mid \boldsymbol{\Gamma}\right]
$$

and

$$
\left[\boldsymbol{Y}_{-t} \mid \boldsymbol{G}^{(T)}\right]=\int\left[\boldsymbol{Y}_{-t} \mid \boldsymbol{B}\right][\boldsymbol{B} \mid \boldsymbol{\Gamma}] \prod_{i, j} \boldsymbol{G}^{(T)}\left(\boldsymbol{\Gamma}_{i j}\right) d \boldsymbol{B} d \boldsymbol{\Gamma}=\int\left[\boldsymbol{Y}_{-t} \mid \boldsymbol{\Gamma}\right] \prod_{i, j} \boldsymbol{G}^{(T)}\left(\boldsymbol{\Gamma}_{i j}\right) d \boldsymbol{\Gamma}
$$

Then the marginal posterior $\left[\boldsymbol{y}_{t} \mid \boldsymbol{Y}_{-t}\right]$ is given by

$$
\left[\boldsymbol{y}_{t} \mid \boldsymbol{Y}_{-t}\right]=\int\left[\boldsymbol{y}_{t} \mid \boldsymbol{G}^{(T)}, \boldsymbol{Y}_{-t}\right] d\left[\boldsymbol{G}^{(T)} \mid \boldsymbol{Y}_{-t}\right]
$$

Note that the marginal posterior $\left[\boldsymbol{G}^{(T)} \mid \boldsymbol{Y}_{-t}\right]$ is an updated discrete distribution of random probability measures. Hence, as in the case of the marginal distribution of $[\boldsymbol{Y}]$, here $\left[\boldsymbol{y}_{t} \mid \boldsymbol{Y}_{-t}\right]$ is a weighted average of models of the form $\left[\boldsymbol{y}_{t} \mid \boldsymbol{G}^{(T)}, \boldsymbol{Y}_{-t}\right]$, the weights being $\left[\boldsymbol{G}^{(T)} \mid \boldsymbol{Y}_{-t}\right]$. 


\section{S-2. Simulation Experiments: Methodology and detailed Results.}

S-2.1. Model comparisons using simulations from cross-validation densities. For all competing models $\mathcal{M}_{j}$, for each $t=1, \ldots, T$, we simulate $N$ realizations $\left\{\tilde{\boldsymbol{y}}_{t}^{(1)}, \ldots, \tilde{\boldsymbol{y}}_{t}^{(N)}\right\}$ from the cross-validation $(\mathrm{CV})$ posterior density

$$
\left[\tilde{\boldsymbol{y}}_{t} \mid \boldsymbol{Y}_{-t}, \mathcal{M}_{j}\right]=\int\left[\tilde{\boldsymbol{y}}_{t} \mid \boldsymbol{\theta}_{j}, \mathcal{M}_{j}\right]\left[\boldsymbol{\theta}_{j} \mid \boldsymbol{Y}_{-t}, \mathcal{M}_{j}\right] d \boldsymbol{\theta}_{j}
$$

where $\tilde{\boldsymbol{y}}_{t}=\left(\tilde{y}_{1}(t), \ldots, \tilde{y}_{R}(t)\right)^{\prime}$ is the random vector corresponding to the observed data vector $\boldsymbol{y}_{t}=\left(y_{1}(t), \ldots, y_{R}(t)\right)^{\prime}$. This exercise can be done by first drawing $N$ realizations $\left\{\boldsymbol{\theta}_{j}^{(1)}, \ldots, \boldsymbol{\theta}_{j}^{(N)}\right\}$ from $\left[\boldsymbol{\theta}_{j} \mid \boldsymbol{Y}_{-t}, \mathcal{M}_{j}\right]$ using our Gibbs sampling algorithm (slightly modified to address the deletion of $\boldsymbol{y}_{t}$ from the entire data set $\boldsymbol{Y}$ ), and then simulating $\tilde{\boldsymbol{y}}_{t}^{(k)}$ from $\left[\tilde{\boldsymbol{y}}_{t} \mid \boldsymbol{\theta}_{j}^{(k)}, \mathcal{M}_{j}\right]$ for $k=1, \ldots, N$. The latter distribution depends upon $\boldsymbol{\theta}_{j}^{(k)}$ only through $\left\{\alpha_{i}^{(k)}, \beta_{i}^{(k)}(t) ; i=1,2, \ldots, R\right\}$ and $\sigma_{\epsilon}^{(k)}$. In practice, rather than simulate repeatedly from $\left[\boldsymbol{\theta}_{j} \mid \boldsymbol{Y}_{-t}, \mathcal{M}_{j}\right]$ for each $t=$ $1, \ldots, T$, (requiring $T=285$ computationally demanding Gibbs sampling runs in our application), we approximate $\left[\boldsymbol{\theta}_{j} \mid \boldsymbol{Y}_{-t}, \mathcal{M}_{j}\right]$ for each $t$ by $\left[\boldsymbol{\theta}_{j} \mid \boldsymbol{Y}, \mathcal{M}_{j}\right]$. This device results in our simulating only one set of MCMC realizations, with substantial computational cost savings.

The 95\% HPD CIs of the marginal cross-validation densities of each of $\tilde{y}_{i}(t) ; i=$ $1, \ldots, R$ are constructed, following Carlin and Louis (1996) from the realizations $\left\{\tilde{\boldsymbol{y}}_{t}^{(1)}, \ldots, \tilde{\boldsymbol{y}}_{t}^{(N)}\right\}$. Then, it is noted whether or not the observed data point $y_{i}(t)$ falls within the corresponding 95\% HPD CI of the CV density of $\tilde{y}_{i}(t)$. We also calculate the length of each $95 \%$ HPD CI. This procedure is repeated for each $t=1, \ldots, T$, and for each $i=1, \ldots, R$, the proportion of observed $\left\{y_{i}(t) ; t=1, \ldots, T\right\}$ falling within the respective $95 \%$ HPD CIs is noted. For each $i$, we also record the mean lengths of these $T=28595 \%$ HPD CIs.

We note that the procedure outlined here is more informative than either the Bayes Factors or its pseudo-version, details of which are provided in Section S2.2. We next provide some details on using cross-validation in the assessment of model adequacy and outlier detection.

S-2.1.1. Posterior predictive p-value. A well-known Bayesian method for assessing goodness-of-fit is the posterior predictive $p$-value (Guttman, 1967; Rubin, 1984; Meng, 1994; Gelman, Meng and Stern, 1996) given by

$$
P\left(V(\tilde{\boldsymbol{Y}})>V(\boldsymbol{Y}) \mid \boldsymbol{Y}, \mathcal{M}_{j}\right)=\int P\left(V(\tilde{\boldsymbol{Y}})>V(\boldsymbol{Y}) \mid \boldsymbol{\theta}_{j}, \mathcal{M}_{j}\right) \pi\left(\boldsymbol{\theta}_{j} \mid \boldsymbol{Y}, \mathcal{M}_{j}\right) d \boldsymbol{\theta}_{j}
$$


where $V(\cdot)$ is any appropriate statistic, $\tilde{\boldsymbol{Y}}=\left\{\tilde{\boldsymbol{y}}_{1}, \ldots, \tilde{\boldsymbol{y}}_{T}\right\}$ is the random variable corresponding to data $\boldsymbol{Y}=\left\{\boldsymbol{y}_{1}, \ldots, \boldsymbol{y}_{T}\right\}$, and

$$
P\left(V(\tilde{\boldsymbol{Y}})>V(\boldsymbol{Y}) \mid \boldsymbol{\theta}_{j}, \mathcal{M}_{j}\right)=\int_{V(\tilde{\boldsymbol{Y}})>V(\boldsymbol{Y})} L\left(\boldsymbol{\theta}_{j}, \tilde{\boldsymbol{Y}} \mid \mathcal{M}_{j}\right) d \tilde{\boldsymbol{Y}}
$$

$L\left(\cdot, \cdot \mid \mathcal{M}_{j}\right)$ denoting the likelihood function corresponding to model $\mathcal{M}_{j}$. The posterior predictive $p$-value (18) is unsatisfactory in the sense that it uses data twice, once to compute the posterior $\pi\left(\boldsymbol{\theta}_{j} \mid \boldsymbol{Y}, \mathcal{M}_{j}\right)$ and then again to compute the tail probability (19) corresponding to $V(\boldsymbol{Y})$ (see Bayarri and Berger, 1999, 2000, who in fact demonstrate that (18) can be over-optimistic in that it does not tend to zero even with overwhelming evidence against the model.) Moreover, (18) does not follow $U(0,1)$ even asymptotically. Apart from this serious disadvantage, the posterior predictive $p$-value does not provide any means for detection of outlying data points even under the assumption that the model $\mathcal{M}_{j}$ is adequate.

$\mathrm{S}$-2.1.2. Advantages of CV-based p-value over posterior predictive p-value. Using approaches based on $\mathrm{CV}$, whether or not a particular data point $\boldsymbol{y}_{t}$ is an outlier with respect to model $\mathcal{M}_{j}$ can be ascertained by computing the $\mathrm{CV} p$-value:

$$
\begin{aligned}
& P\left(V\left(\tilde{\boldsymbol{y}}_{t}\right)>V\left(\boldsymbol{y}_{t}\right) \mid \boldsymbol{Y}_{-t}, \mathcal{M}_{j}\right) \\
&=\int P\left(V\left(\tilde{\boldsymbol{y}}_{t}\right)>V\left(\boldsymbol{y}_{t}\right) \mid \boldsymbol{\theta}_{j}, \mathcal{M}_{j}\right) \pi\left(\boldsymbol{\theta}_{j} \mid \boldsymbol{Y}_{-t}, \mathcal{M}_{j}\right) d \boldsymbol{\theta}_{j},
\end{aligned}
$$

where

$$
P\left(V\left(\tilde{\boldsymbol{y}}_{t}\right)>V\left(\boldsymbol{y}_{t}\right) \mid \boldsymbol{\theta}_{j}, \mathcal{M}_{j}\right)=\int_{V\left(\tilde{\boldsymbol{y}}_{t}\right)>V\left(\boldsymbol{y}_{t}\right)} L\left(\boldsymbol{\theta}_{j}, \tilde{\boldsymbol{y}}_{t} \mid \mathcal{M}_{j}\right) d \tilde{\boldsymbol{y}}_{t}
$$

Assuming adequacy of the model $\mathcal{M}_{j}$, a small value of (20) indicates that $\boldsymbol{y}_{t}$ is an outlier. However, if all (or most of) the $\mathrm{CV} p$-values, corresponding to $\left\{\boldsymbol{y}_{1}, \ldots, \boldsymbol{y}_{T}\right\}$ are small, then inadequacy of model $\mathcal{M}_{j}$ is indicated.

In contrast with posterior predictive $p$-value, (20) avoids double use of the data, since $\boldsymbol{Y}_{-t}$ is used to compute the posterior $\pi\left(\boldsymbol{\theta} \mid \boldsymbol{Y}_{-t}, \mathcal{M}_{j}\right)$, while $\boldsymbol{y}_{t}$, the data point left out, is involved in the computation of (21). Also, conditional on $\boldsymbol{Y}_{-t}$, (20) is just the complement of the distribution function $F\left(V(\cdot) \mid \boldsymbol{Y}_{-t}\right)$, and hence follows $U(0,1)$. Thus, the tail probabilities based on the CV posteriors $\pi\left(\tilde{\boldsymbol{y}}_{t} \mid\right.$ $\left.\boldsymbol{Y}_{-t}\right)$ are correctly estimated and not overoptimistic, unlike the posterior predictive $p$-values.

S-2.2. Other methods of model comparison. In this section, we discuss Bayes Factors (BF), their pseudo- versions, and their shortcomings in the context of model comparisons. 
S-2.2.1. Bayes Factors. Let $\boldsymbol{y}_{t}=\left(y_{1}(t), \ldots, y_{R}(t)\right)^{\prime}$ denote the observation data vector, and let $\boldsymbol{Y}=\left\{\boldsymbol{y}_{1}, \ldots, \boldsymbol{y}_{T}\right\}$. The BF for comparing two models $\mathcal{M}_{1}$ and $\mathcal{M}_{2}$, is given by $B F\left(\mathcal{M}_{1} / \mathcal{M}_{2}\right)=\frac{\left[\boldsymbol{Y} \mid \mathcal{M}_{1}\right]}{\left[\boldsymbol{Y} \mid \mathcal{M}_{2}\right]}$, where, for $j=1,2,\left[\boldsymbol{Y} \mid \mathcal{M}_{j}\right]=$ $\int\left[\boldsymbol{Y} \mid \boldsymbol{\theta}_{j}, \mathcal{M}_{j}\right]\left[\boldsymbol{\theta}_{j} \mid \mathcal{M}_{j}\right] d \boldsymbol{\theta}_{j}$ is the marginal distribution of $\boldsymbol{Y}$ under $\mathcal{M}_{j}$, with corresponding parameter set $\boldsymbol{\theta}_{j}$. BFs are not particularly appealing here however, because they have a tendency to put excessive weight on parsimonious models. This phenomenon is known as Lindley's paradox - see, e.g. Bartlett (1957) and also Gelfand and Dey (1994) who prove this formally under suitable regularity conditions. This complicates matters because our goal is to investigate utility of the substantially more complex $\mathcal{M}_{D P}$ relative to the simpler $\mathcal{M}_{R W}$ and $\mathcal{M}_{A R}$. Further, with improper priors the marginal density of the data is improper. Numerical methods for computing the BF (Kass and Raftery, 1995) are also unsatisfactory for highly structured and high-dimensional models, such as those considered in this paper.

S-2.2.2. Pseudo-Bayes Factors. Pseudo-Bayes factors (PBF) are defined for two competing models $\mathcal{M}_{1}$ and $\mathcal{M}_{2}$, as $\operatorname{PBF}\left(\mathcal{M}_{1} / \mathcal{M}_{2}\right)=\prod_{t=1}^{T} \frac{\left[\boldsymbol{y}_{t} \mid \boldsymbol{Y}_{-t}, \mathcal{M}_{1}\right]}{\left[\boldsymbol{y}_{t} \mid \boldsymbol{Y}_{-t}, \mathcal{M}_{2}\right]}$, where $\boldsymbol{Y}_{-t}=\left\{\boldsymbol{y}_{1}, \ldots, \boldsymbol{y}_{t-1}, \boldsymbol{y}_{t+1}, \ldots, \boldsymbol{y}_{T}\right\}$, for $T>1$ (Geisser and Eddy, 1979). By Brook (1964)'s lemma, the set of cross-validation densities $\left[\boldsymbol{y}_{t} \mid \boldsymbol{Y}_{-t}, \mathcal{M}_{j}\right]$ is equivalent to the marginal density $\left[\boldsymbol{Y} \mid \mathcal{M}_{j}\right]$ for any model $\mathcal{M}_{j}$, provided that it exists. Thus, exactly the same information is utilized for computing BF and PBF, but the latter avoids Lindley's paradox (see e.g. Gelfand and Dey, 1994, for a formal proof under appropriate regularity conditions). Also, the cross-validation densities are proper whenever the posteriors of the parameters given $\boldsymbol{Y}_{-t}$ are proper. Computationally also, PBFs seem more stable, since each cross-validation density $\left[\boldsymbol{y}_{t} \mid \boldsymbol{Y}_{-t}, \mathcal{M}_{j}\right] ; j=1,2, t=1, \ldots, T$, is a function in $\boldsymbol{y}_{t}$ only, which is very low-dimensional. However, the time taken to compute $T$ cross-validation densities can be excessive for large $T$. Indeed,

$$
\left[\boldsymbol{y}_{t} \mid \boldsymbol{Y}_{-t}, \mathcal{M}_{j}\right]=\int\left[\boldsymbol{y}_{t} \mid \boldsymbol{\theta}_{j}, \mathcal{M}_{j}\right]\left[\boldsymbol{\theta}_{j} \mid \boldsymbol{Y}_{-t}, \mathcal{M}_{j}\right] d \boldsymbol{\theta}_{j} \approx \frac{1}{N} \sum_{\ell=1}^{N}\left[\boldsymbol{y}_{t} \mid \boldsymbol{\theta}_{j}^{(\ell)}\right]
$$

where $\left\{\boldsymbol{\theta}_{j}^{(\ell)} ; \ell=1, \ldots, N\right\}$ is a set of MCMC simulations from the full conditional posterior distribution of (the set of) model parameters $\boldsymbol{\theta}_{j}$, denoted by $\left[\boldsymbol{\theta}_{j} \mid \boldsymbol{Y}_{-t}, \mathcal{M}_{j}\right]$. Hence, for each $t=1, \ldots, T$, a separate MCMC algorithm is needed to simulate from the posterior density $\left[\boldsymbol{\theta}_{j} \mid \boldsymbol{Y}_{-t}, \mathcal{M}_{j}\right]$. In our application, $T=285$, so 285 separate MCMC algorithms are necessary. Importance-samplingbased approximations proposed by Gelfand (1996) to alleviate this problem have the potential to provide poor approximations in high dimensions (see e.g. Peruggia, 
1997). For large data sets such as ours, the approximation $\left[\boldsymbol{\theta}_{j} \mid \boldsymbol{Y}_{-t}, \mathcal{M}_{j}\right] \approx\left[\boldsymbol{\theta}_{j} \mid\right.$ $\left.\boldsymbol{Y}, \mathcal{M}_{j}\right] \quad \forall t$ is commonly used and quite accurate (see e.g. Gelfand, 1996).

However, if $\frac{\left[\boldsymbol{y}_{k} \mid \boldsymbol{Y}_{-k}, \mathcal{M}_{1}\right]}{\left[\boldsymbol{y}_{k} \mid \boldsymbol{Y}_{-k}, \mathcal{M}_{2}\right]} \approx 0$ for some $k \in\{1, \ldots, T\}$, then $P B F\left(\mathcal{M}_{1} / \mathcal{M}_{2}\right) \approx$ 0 , even if $\left[\boldsymbol{y}_{t} \mid \boldsymbol{Y}_{-t}, \mathcal{M}_{1}\right]>\left[\boldsymbol{y}_{t} \mid \boldsymbol{Y}_{-t}, \mathcal{M}_{2}\right]$ for all $t \neq k$. This means that a single data point $\boldsymbol{y}_{k}$ acts as an extreme outlier for model $\mathcal{M}_{1}$; even though $\mathcal{M}_{1}$ outperforms $\mathcal{M}_{2}$ for all other data points, emerging as the better model, the single data point $\boldsymbol{y}_{k}$ forces PBF to select $\mathcal{M}_{2}$. Thus, a single observation can have much influence on model selection by PBF, since only density at the observed points are used, pointing to a serious issue with PBFs.

S-2.3. Results of simulation study. Figures S-1 and S-2 display the marginal posterior distributions of $\gamma_{i j}(t)$ obtained using $\mathcal{M}_{A R}$ and $\mathcal{M}_{D P}$, respectively. Observe that $\mathcal{M}_{A R}$ performs better than the $\mathcal{M}_{R W}$ of Figure 1 (of the paper) but model $\mathcal{M}_{D P}$ is the winner. The support of the posterior distributions of $\gamma_{22}(t)$ and $\gamma_{23}(t)$ using $\mathcal{M}_{A R}$ are too wide to be of much use, but quite adequate under

TABLE S-1

Proportion of true $\gamma_{i j}(t)$ included in the corresponding $95 \%$ HPD credible intervals obtained using $\mathcal{M}_{A R}$ and $\mathcal{M}_{D P}$ on data simulated under $\mathcal{M}_{R W^{\prime}}$.

\begin{tabular}{|c|c|c|c|c|c|c|c|c|c|}
\hline Model & $\gamma_{11}$ & $\gamma_{12}$ & $\gamma_{13}$ & $\gamma_{21}$ & $\gamma_{22}$ & $\gamma_{23}$ & $\gamma_{31}$ & $\gamma_{32}$ & $\gamma_{33}$ \\
\hline $\mathcal{M}_{A R}$ & 0.89 & 0.99 & 0.91 & 0.95 & 1.0 & 1.0 & 0.73 & 0.66 & 0.38 \\
\hline $\mathcal{M}_{D P}$ & 1.0 & 0.99 & 0.99 & 1.0 & 1.0 & 1.0 & 0.99 & 0.93 & 0.99 \\
\hline
\end{tabular}

$\mathcal{M}_{D P}$. Note that the large posterior variabilities $\gamma_{i j}(t)$ at some large values of $t$ are not unexpected for $\mathcal{M}_{R W}$ and $\mathcal{M}_{A R}$ tending towards $\mathcal{M}_{R W}$, since the prior variances of $\gamma_{i j}(t) \mathrm{s}$ increase to infinity with $t$ in both cases. This, through equation (2) of the paper, significantly inflates the variance of the data. Such issues are avoided in $\mathcal{M}_{D P}$, making it a better candidate compared to $\mathcal{M}_{R W}$ and $\mathcal{M}_{A R}$. These observations are further validated by Table $S-8$ which provides proportions of true $\gamma_{i j}(t)$ 's that are included in the corresponding 95\% HPD credible intervals. Both Tables 1 (in the paper) and S-8 show that $\mathcal{M}_{D P}$ outperforms the other two models. This is as expected because $\mathcal{M}_{D P}$ best quantifies model uncertainty for $\gamma_{i j}(t)$.

Figure S-3 shows the posterior distributions of $\rho$ under $\mathcal{M}_{A R}$ and $\mathcal{M}_{D P}$. The posterior of $\rho$ under $\mathcal{M}_{D P}$ has much wider support than that using $\mathcal{M}_{A R}$. This is a consequence of the flexibility inherent in the DP-based methodology, which also ensures that the $95 \%$ HPD credible intervals under $\mathcal{M}_{D P}$ capture almost all of the true values of $\gamma_{i j}(t)$. Comparatively lesser coverage under $\mathcal{M}_{A R}$ leads to non-inclusion of some true values of $\gamma_{i j}(t)$ in their respective 95\% HPD credible intervals.

Model $\mathcal{M}_{D P}$ also exhibited better predictive performance than $\mathcal{M}_{A R}$ and $\mathcal{M}_{R W}$ in the sense that almost all the observed data points $y_{i}(t) ; i=1,2,3 ; t=1, \ldots, T$ fell in the $95 \%$ credible intervals of the respective posterior predictive densities, and 


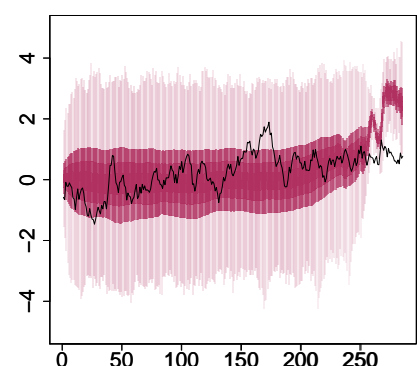

(a) $\gamma_{11}(t)$

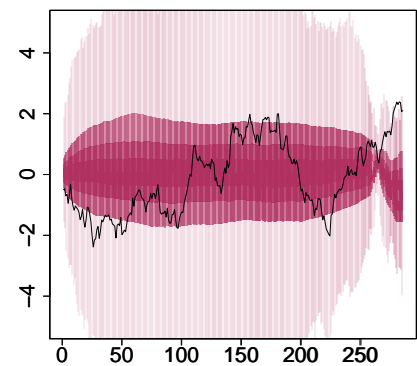

(d) $\gamma_{21}(t)$

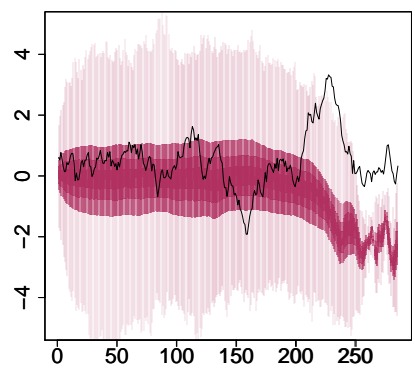

(g) $\gamma_{31}(t)$

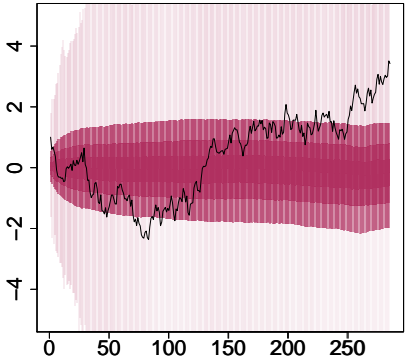

(b) $\gamma_{12}(t)$

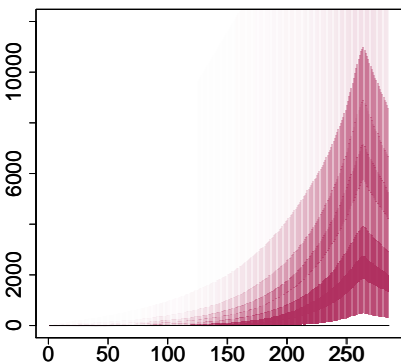

(e) $\gamma_{22}(t)$

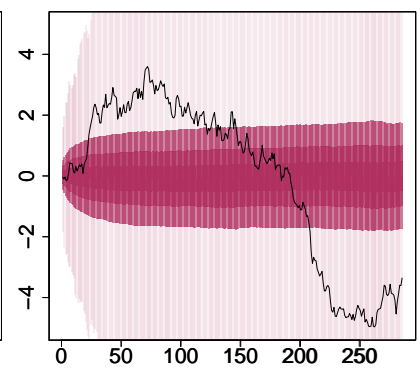

(h) $\gamma_{32}(t)$

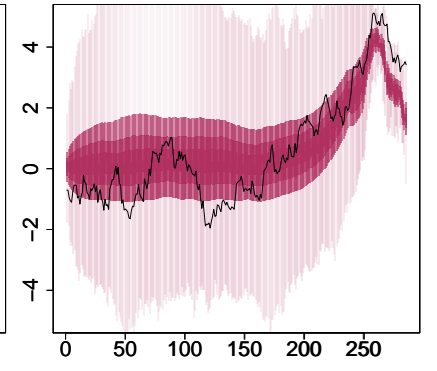

(c) $\gamma_{13}(t)$

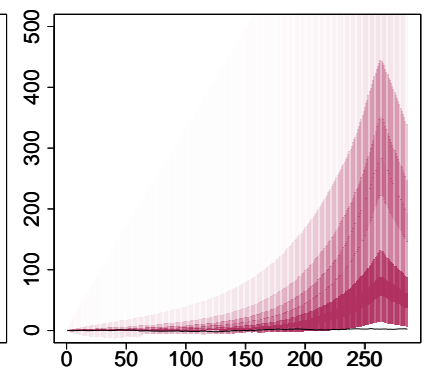

(f) $\gamma_{23}(t)$

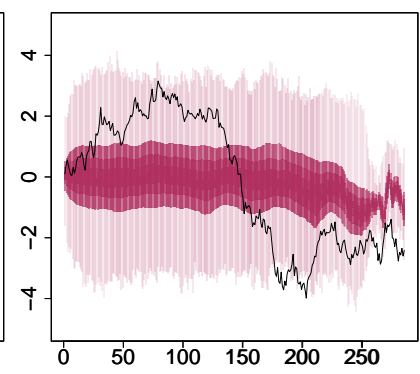

(i) $\gamma_{33}(t)$

FIG S-1. Simulation study: Posterior densities of $\gamma_{i j}(t) ; t=1, \ldots, T ; i, j=1,2,3$ with respect to the stationary AR(1) modeling of $\gamma_{i j}$. Displays are as in Figure 1 of the paper.

the average length of such credible intervals was the smallest under $\mathcal{M}_{D P}$ (Table S-2). Note that we refer to the average of the lengths of the $95 \%$ credible intervals of as the average length of the $95 \%$ credible interval of $y_{i}$, for $i=1,2,3$ in Table S2. All these results, which speak in favor of our DP-based model, are implicitly the consequence of the fact that the true model is approximately non-stationary, and is modeled more flexibly by our non-stationary DP model rather than the stationary AR(1) model. That this borderline between stationarity and non-stationarity of the true model is important was vindicated by another simulation experiment we performed, where the data were drawn from $\mathcal{M}_{R W}$ but $\mathcal{M}_{R W}, \mathcal{M}_{A R}$ and $\mathcal{M}_{D P}$ were 


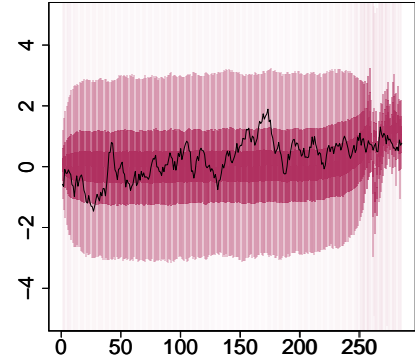

(a) $\gamma_{11}(t)$

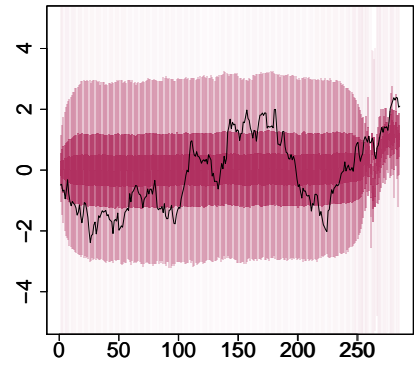

(d) $\gamma_{21}(t)$

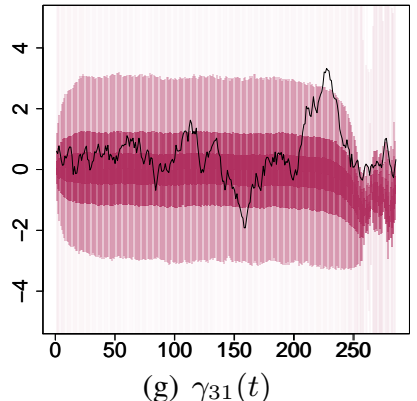

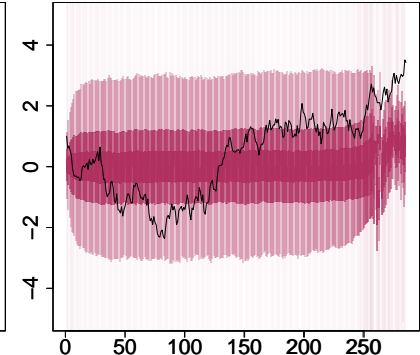

(b) $\gamma_{12}(t)$

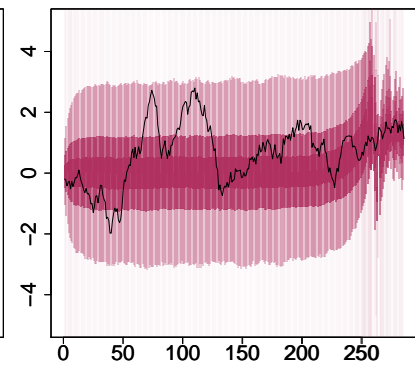

(e) $\gamma_{22}(t)$

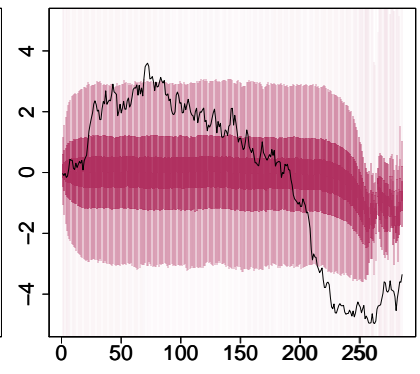

(h) $\gamma_{32}(t)$

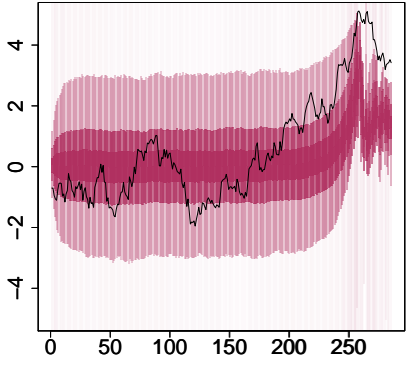

(c) $\gamma_{13}(t)$

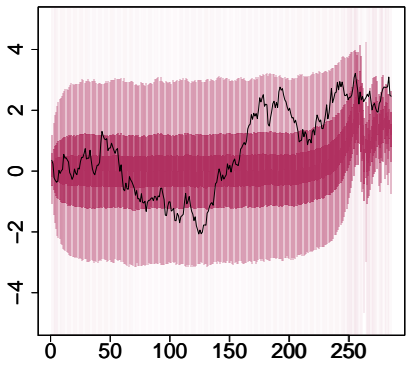

(f) $\gamma_{23}(t)$

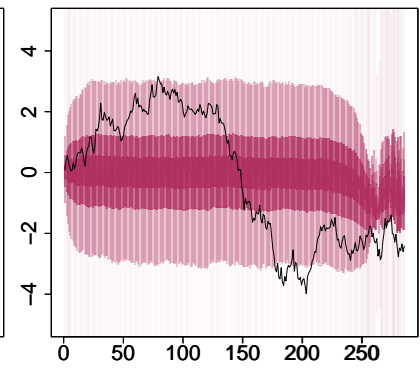

(i) $\gamma_{33}(t)$

FIG S-2. Simulation study: Posterior densities of $\gamma_{i j}(t) ; t=1, \ldots, T ; i, j=1,2,3$ with respect to the Dirichlet process modeling of $\gamma_{i j}$. Displays are as in Figure 1 of the paper.

each used to fit the data. In this case, $\mathcal{M}_{R W}$ outperformed both $\mathcal{M}_{D P}$ and $\mathcal{M}_{A R}$ in terms of coverage of the true values of $\gamma_{i j}(t)$ (Table S-5), indicating that $\mathcal{M}_{D P}$ may under-perform when compared to the true model, in terms of coverage of parameter values, when the true model can be clearly identified. However, Table S-4 shows that in terms of prediction ability measured here in terms of coverage of the data points $y_{i}(t)$ and lengths of the associated $95 \%$ credible intervals, $\mathcal{M}_{D P}$ is still the best performer. This is not unexpected, since $\mathcal{M}_{D P}$ involves model averaging, which improves predictive performance (see, e.g., Kass and Raftery, 1995). 


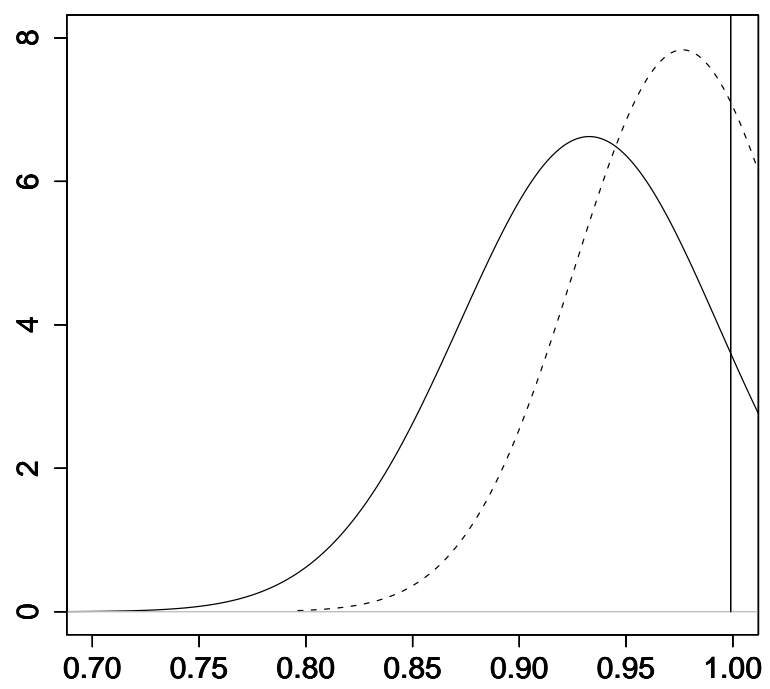

FIG S-3. Posterior distribution of $\rho$ using $\mathcal{M}_{A R}$ (broken line) and $\mathcal{M}_{D P}$ (solid line). The vertical solid line indicates the true value $(\rho=0.999$.

TABLE S-2

Proportions of observed data included in the $95 \%$ credible intervals of the corresponding posterior predictive distributions and their mean lengths upon fitting $\mathcal{M}_{R W}, \mathcal{M}_{A R}$ and $\mathcal{M}_{D P}$ and using data simulated under $\mathcal{M}_{R W^{\prime}}$.

\begin{tabular}{|c|c|c|c|c|c|c|}
\hline \multirow[t]{2}{*}{$y$} & \multicolumn{3}{|c|}{ Proportion } & \multicolumn{3}{|c|}{ Mean Length $\left(\times 10^{9}\right)$} \\
\hline & $\mathcal{M}_{R W}$ & $\mathcal{M}_{A R}$ & $\mathcal{M}_{D P}$ & $\mathcal{M}_{R W}$ & $\mathcal{M}_{A R}$ & $\mathcal{M}_{D P}$ \\
\hline$y$ & 1.0 & 1.0 & 1.0 & 1.238 & 1.033 & 0.244 \\
\hline$y$ & 0.94 & 1.00 & 0.99 & 1.169 & 0.992 & 0.244 \\
\hline & 0.97 & 1.00 & 1.00 & 1.192 & 1.005 & 0.242 \\
\hline
\end{tabular}

S-2.3.1. Additional Experiments. So far, we have compared performance using $\mathcal{M}_{D P}, \mathcal{M}_{A R}$ and $\mathcal{M}_{R W}$ on two simulation datasets drawn from $\mathcal{M}_{R W^{\prime}}$ and $\mathcal{M}_{R W}$. Model $\mathcal{M}_{R W^{\prime}}$ was actually a stationary $\operatorname{AR}(1)$ model $\mathcal{M}_{A R}$ with $\rho=$ 0.999 i.e. a model very close to non-stationarity. Here, $\mathcal{M}_{D P}$ was the best performer among the three competitors whether in terms of coverage of the true values of the $\gamma_{i j}$ s, coverage of the observed data, and the associated lengths of the respective $95 \%$ HPD credible intervals. When data were simulated from $\mathcal{M}_{R W}$, $\mathcal{M}_{R W}$ performed marginally better than $\mathcal{M}_{D P}$ and $\mathcal{M}_{A R}$ in terms of coverage of the true values of the $\gamma_{i j}$ 's but both $\mathcal{M}_{R W}$ and $\mathcal{M}_{A R}$ were outperformed by $\mathcal{M}_{D P}$ in terms of coverage of the observed data and the lengths of the respective $95 \%$ HPD credible intervals. 
TABLE S-3

Proportion of true $\gamma_{i j}(t)$ included in the corresponding $95 \%$ HPD credible intervals obtained using $\mathcal{M}_{R W}, \mathcal{M}_{A R}$ and $\mathcal{M}_{D P}$ on data simulated under $\mathcal{M}_{R W}$.

\begin{tabular}{|c|c|c|c|c|c|c|c|c|c|}
\hline Model & $\gamma_{11}$ & $\gamma_{12}$ & $\gamma_{13}$ & $\gamma_{21}$ & $\gamma_{22}$ & $\gamma_{23}$ & $\gamma_{31}$ & $\gamma_{32}$ & $\gamma_{33}$ \\
\hline $\mathcal{M}_{R W}$ & 0.91 & 0.99 & 0.84 & 0.91 & 1.0 & 1.0 & 0.88 & 1.0 & 0.38 \\
\hline $\mathcal{M}_{A R}$ & 0.81 & 0.96 & 0.30 & 0.91 & 1.0 & 1.0 & 0.56 & 1.0 & 0.59 \\
\hline $\mathcal{M}_{D P}$ & 0.93 & 0.43 & 0.64 & 0.73 & 1.0 & 0.96 & 0.94 & 0.47 & 0.45 \\
\hline
\end{tabular}

TABLE S-4

Proportions of observed data included in the $95 \%$ credible intervals of the corresponding posterior predictive distributions and their mean lengths upon fitting $\mathcal{M}_{R W}, \mathcal{M}_{A R}$ and $\mathcal{M}_{D P}$ and using data simulated under $\mathcal{M}_{R W}$.

\begin{tabular}{|c||ccc||ccc|}
\hline \multicolumn{1}{|c||}{$y$} & \multicolumn{3}{c||}{ Proportion } & \multicolumn{3}{c|}{ Mean Length $\left(\times 10^{7}\right)$} \\
& $\mathcal{M}_{R W}$ & $\mathcal{M}_{A R}$ & $\mathcal{M}_{D P}$ & $\mathcal{M}_{R W}$ & $\mathcal{M}_{A R}$ & $\mathcal{M}_{D P}$ \\
\hline$y_{1}$ & 1.0 & 1.0 & 1.0 & 5.568 & 4.471 & 1.967 \\
$y_{2}$ & 0.94 & 0.93 & 1.0 & 4.531 & 3.952 & 1.992 \\
$y_{3}$ & 0.99 & 0.96 & 1.0 & 4.778 & 4.063 & 1.958 \\
\hline
\end{tabular}

The simulation studies described so far may lead one to expect that when the data is close to non-stationarity $\mathcal{M}_{D P}$ performs best among all the three models with respect to either or both the criteria of coverage of the true $\gamma_{i j}$ 's and the observed data and lengths of their respective 95\% HPD credible intervals. It is also reasonable to anticipate that when the data is actually (asymptotically) stationary and is clearly distinguishable from non-stationarity (even asymptotically), then $\mathcal{M}_{D P}$ will fail to perform as well as $\mathcal{M}_{A R}$. We investigate here with two additional simulation studies, whether such expectations are met.

The two data sets were both simulated from $\mathcal{M}_{A R}$ but one had $\rho=0.5$ and the other set $\rho=0.95$. Thus, we had a simulated dataset that is clearly stationary ( $\rho=$ $0.5)$ and one that is less clearly distinguished from non-stationarity $(\rho=0.95)$. Table S-5 shows the proportions of true $\gamma_{i j}$ 's included in the respective 95\% HPD credible intervals under the three models for each of the two data sets. Clearly, for $\rho=0.5, \mathcal{M}_{A R}$ outperforms $\mathcal{M}_{D P}$ marginally and $\mathcal{M}_{R W}$ by a significant margin. Note that as far as predicting the observed data is concerned, $\mathcal{M}_{A R}$ betters the performance of $\mathcal{M}_{D P}$ and $\mathcal{M}_{R W}$ in terms of the proportions of the observed data included in their respective 95\% HPD credible intervals, but the lengths of the $95 \%$ HPD credible intervals with respect to $\mathcal{M}_{R W}$ are less than those of $\mathcal{M}_{A R}$; see Table S-6. The latter problem is not of particular significance however, since lengths of the credible intervals are important only when it is ensured that two models perform equally well in terms of inclusion of the observed data in the respective credible intervals.

Thus we see that in the case of $\rho=0.95, \mathcal{M}_{D P}$ performs better than $\mathcal{M}_{A R}$ and $\mathcal{M}_{R W}$ in terms of both the criteria of inclusion proportions of $\gamma_{i j}$ 's and inclusion 
TABLE S-5

Proportion of true $\gamma_{i j}(t)$ included in the corresponding $95 \%$ HPD credible intervals obtained using $\mathcal{M}_{R W}, \mathcal{M}_{A R}$ and $\mathcal{M}_{D P}$ on data simulated under $\mathcal{M}_{A R}$ with $\rho=0.5$ and $\rho=0.95$.

\begin{tabular}{|c||c|c|c|c|c|c|c|c|c|c|}
\hline True AR Model & Fitted Model & $\gamma_{11}$ & $\gamma_{12}$ & $\gamma_{13}$ & $\gamma_{21}$ & $\gamma_{22}$ & $\gamma_{23}$ & $\gamma_{31}$ & $\gamma_{32}$ & $\gamma_{33}$ \\
\hline \multirow{3}{*}{$\rho=0.5$} & $\mathcal{M}_{R W}$ & 1.0 & 1.0 & 0.91 & 1.0 & 0 & 0.05 & 0.04 & 1.0 & 0 \\
\cline { 2 - 11 } & $\mathcal{M}_{A R}$ & 0.30 & 1.0 & 1.0 & 1.0 & 1.0 & 1.0 & 0.97 & 1.0 & 1.0 \\
\cline { 2 - 10 } & $\mathcal{M}_{D P}$ & 0.24 & 1.0 & 1.0 & 0.96 & 1.0 & 1.0 & 0.96 & 1.0 & 1.0 \\
\hline \multirow{3}{*}{$\rho=0.95$} & $\mathcal{M}_{R W}$ & 0.4 & 1.0 & 0.99 & 1.0 & 1.0 & 1.0 & 0.74 & 1.0 & 1.0 \\
\cline { 2 - 10 } & $\mathcal{M}_{A R}$ & 0.48 & 0.99 & 1.0 & 1.0 & 1.0 & 1.0 & 0.78 & 1.0 & 1.0 \\
\cline { 2 - 10 } & $\mathcal{M}_{D P}$ & 0.74 & 0.99 & 1.0 & 0.84 & 1.0 & 0.99 & 0.82 & 0.99 & 0.88 \\
\hline
\end{tabular}

TABLE S-6

Proportions of observed data included in the 95\% credible intervals of the corresponding posterior predictive distributions and their mean lengths upon fitting $\mathcal{M}_{R W}, \mathcal{M}_{A R}$ and $\mathcal{M}_{D P}$ and using data simulated under $\mathcal{M}_{A R}$ with $\rho=0.5$.

\begin{tabular}{|c||ccc|ccc|}
\hline \multicolumn{1}{|c||}{} & \multicolumn{5}{c|}{ True Model: $\mathcal{M}_{A R}$ with $\rho=0.5$} \\
\cline { 2 - 7 }$y$ & \multicolumn{3}{c|}{ Proportion } & \multicolumn{3}{c|}{ Mean Length } \\
& $\mathcal{M}_{R W}$ & $\mathcal{M}_{A R}$ & $\mathcal{M}_{D P}$ & $\mathcal{M}_{R W}$ & $\mathcal{M}_{A R}$ & $\mathcal{M}_{D P}$ \\
\hline$y_{1}$ & 0.97 & 0.99 & 0.98 & 818.3 & 844.1 & 902.6 \\
$y_{2}$ & 0.96 & 0.99 & 0.99 & 824.6 & 843.4 & 904.6 \\
$y_{3}$ & 0.96 & 0.99 & 0.99 & 820.9 & 843.4 & 898.0 \\
\hline
\end{tabular}

proportions of the data, along with the lengths of the respective 95\% HPD credible intervals. The details are shown in Tables S-5, S-6, and S-7. The results of the simulation studies described here are in agreement with what is expected of the performance of our model and methodology, and once again demonstrate the advantage of the non-stationary DP-based modeling in situations where stationarity of the data set can not be clearly established.

\section{S-2.4. Additional Details on Simulation Experiments.}

S-2.4.1. Sensitivity analysis with respect to the prior on $\tau$. Our analysis done using model $\mathcal{M}_{D P}$ involves specifying a $\Gamma\left(a_{\tau}, b_{\tau}\right)$ prior on $\tau$ where $a_{\tau}$ and $b_{\tau}$ both depend on the choice of $c$. We used $c=0.1$ in all our experiments to reflect large variance for our prior distribution. We evaluated sensitivity of obtained results to $c$ by also using two other choices of $c=0.001$ and $c=0.01$ when fitting $\mathcal{M}_{D P}$ to data simulated under model $\mathcal{M}_{R W^{\prime}}$. Tables S-8 and S-9 provide comparative summaries of these results. We see that the inclusion percentages of the true values of $\gamma_{i j}(t) \mathrm{s}$ are quite robust but for $\gamma_{32}(t)$ and $\gamma_{33}(t)$. However, $\mathcal{M}_{D P}$ with $c=0.1$ is the best performer, having the smallest average mean length of the 95\% HPD predictive intervals. Even then, these intervals are very substantially shorter than those obtained using $\mathcal{M}_{A R}$ and $\mathcal{M}_{R W}$. 
TABLE S-7

Proportions of observed data included in the $95 \%$ credible intervals of the corresponding posterior predictive distributions and their mean lengths upon fitting $\mathcal{M}_{R W}, \mathcal{M}_{A R}$ and $\mathcal{M}_{D P}$ and using data simulated under $\mathcal{M}_{A R}$ with $\rho=0.95$.

\begin{tabular}{|c||ccc|ccc|}
\hline \multicolumn{1}{|c||}{} & \multicolumn{5}{c|}{ True Model: $\mathcal{M}_{A R}$ with $\rho=0.95$} \\
\cline { 2 - 7 }$y$ & \multicolumn{3}{c|}{ Proportion } & \multicolumn{3}{c|}{ Mean Length } \\
& $\mathcal{M}_{R W}$ & $\mathcal{M}_{A R}$ & $\mathcal{M}_{D P}$ & $\mathcal{M}_{R W}$ & $\mathcal{M}_{A R}$ & $\mathcal{M}_{D P}$ \\
\hline$y_{1}$ & 0.92 & 0.92 & 1.0 & 5,147 & 4,989 & 3,503 \\
$y_{2}$ & 1.0 & 1.0 & 1.0 & 3,842 & 3,728 & 3,067 \\
$y_{3}$ & 1.0 & 1.0 & 1.0 & 4,501 & 4,451 & 3,202 \\
\hline
\end{tabular}

TABLE S-8

Proportion of true $\gamma_{i j}(t)$ included in the corresponding $95 \%$ HPD credible intervals obtained using $\mathcal{M}_{D P}$ with $c=0.001,0.01,0.1$ on data simulated under $\mathcal{M}_{R W^{\prime}}$.

\begin{tabular}{|c|c|c|c|c|c|c|c|c|c|}
\hline$c$ & $\gamma_{11}$ & $\gamma_{12}$ & $\gamma_{13}$ & $\gamma_{21}$ & $\gamma_{22}$ & $\gamma_{23}$ & $\gamma_{31}$ & $\gamma_{32}$ & $\gamma_{33}$ \\
\hline 0.001 & 0.99 & 0.86 & 0.80 & 0.89 & 0.88 & 0.82 & 0.87 & 0.38 & 0.40 \\
\hline 0.01 & 1.00 & 0.94 & 0.90 & 0.95 & 0.90 & 0.89 & 0.88 & 0.42 & 0.51 \\
\hline 0.1 & 1.0 & 0.99 & 0.99 & 1.0 & 1.0 & 1.0 & 0.99 & 0.93 & 0.99 \\
\hline
\end{tabular}

\section{S-3. Additional Details on Analysis of Stroop Task Data.}

S-3.1. The Dataset. Figure S-4 provides a graphical display of the modeled BOLD response $x(t)$ and the three detrended time series $y_{1}(t), y_{2}(t)$ and $y_{3}(t)$ (measured BOLD responses) obtained after pre-processing the Stroop Task dataset.

\section{S-3.2. Convergence of the MCMC algorithms.}

S-3.2.1. Unrestricted model $\mathcal{M}_{D P}$. Convergence assessment of our methodologygenerated MCMC samples using informal tools such as simple trace plots, autocorrelation plots, etc., showed no evidence of non-convergence of the MCMC samples of the unknown parameters. Table S-10 summarizes the estimated autocorrelation functions (ACF) and the Monte Carlo standard errors of the posterior distributions of the unknown parameters in $\mathcal{M}_{D P}$. We do not find much evidence of lack of convergence in the MCMC samples. To confirm this more formally at least in the case

TABLE S-9

Proportions of observed data included in the $95 \%$ credible intervals of the corresponding posterior predictive distributions and their mean lengths upon fitting $\mathcal{M}_{R W}, \mathcal{M}_{A R}$ and $\mathcal{M}_{D P}$ and using data simulated under $\mathcal{M}_{R W^{\prime}}$.

\begin{tabular}{|c||ccc||ccc|}
\hline \multicolumn{1}{|c||}{$y$} & \multicolumn{3}{c||}{ Proportion } & \multicolumn{3}{c|}{ Mean Length $\left(\times 10^{9}\right)$} \\
& $c=0.001$ & $c=0.01$ & $c=0.1$ & $c=0.001$ & $c=0.01$ & $c=0.1$ \\
\hline$y_{1}$ & 0.99 & 1.00 & 1.0 & 0.360 & 0.372 & 0.244 \\
$y_{2}$ & 1.00 & 1.00 & 0.99 & 0.363 & 0.373 & 0.244 \\
$y_{3}$ & 1.00 & 1.00 & 1.00 & 0.360 & 0.371 & 0.242 \\
\hline
\end{tabular}




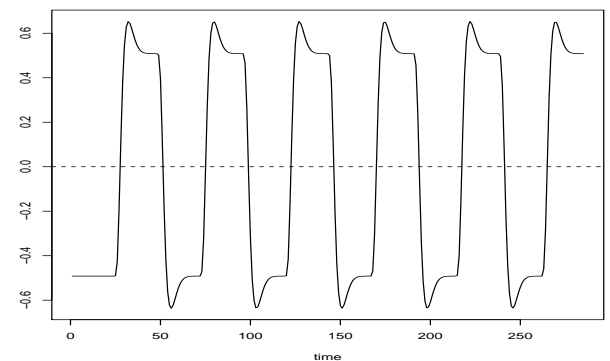

(a) $x(t)$

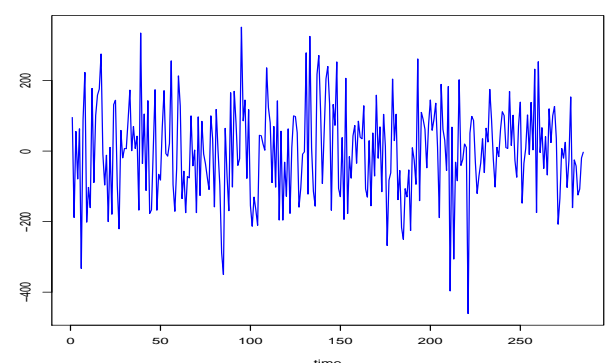

(c) $y_{2}(t)$

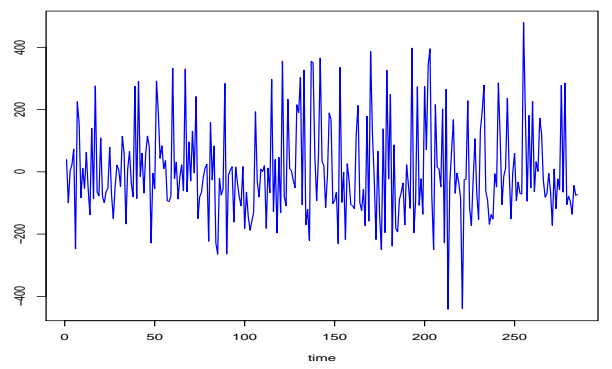

(b) $y_{1}(t)$

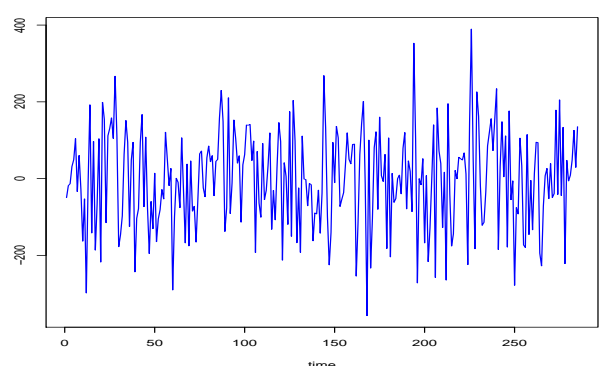

(d) $y_{3}(t)$

FIG S-4. (a) The modeled BOLD response $x(t)$ and the detrended time series (measured BOLD responses) obtained over time for the (b) LG, (c) MOG and (d) DLPFC regions.

of $\gamma_{i j}(t)$ 's, the parameters of interest, we adopted the Kolmogorov-Smirnov (KS) test to diagnose lack of convergence of the chains corresponding to the individual components of the parameters. We refer to Robert and Casella (2004) pp. 466-470 for details, noting in brief that if samples $\left\{\theta^{(1)}, \ldots, \theta^{(N)}\right\}$ are available for estimating the posterior distribution of any parameter component $\theta$ (say), the distributions of the two subsamples $\left\{\theta^{(1)}, \ldots, \theta^{(N / 2)}\right\}$ and $\left\{\theta^{(N / 2+1)}, \ldots, \theta^{(N)}\right\}$ may be compared using the Kolmogorov-Smirnov (K-S) test. Small $p$-values indicate that the two subsamples come from different distributions, pointing to lack of convergence of the MCMC samples to the target posterior (stationary) distribution. K-S tests however require independent samples but our realizations are from a (dependent) Markov Chain. We therefore, made our samples approximately independent by using a thinned sample, using realizations corresponding to every 25 th iteration after burn-in. (Note that we used the unthinned sample after burn-in for other inferential purposes, since they do not require independence). Figure S-5 displays the obtained $p$-values and shows that a vast majority of them are far away from zero. Having calculated the $p$-values of K-S tests conducted for each of the parameters 
TABLE S-10

Stroop task data analysis: Convergence details of the unknown variables associated with the unrestricted DP model $\mathcal{M}_{D P}$.

\begin{tabular}{|c|ccccc|}
\hline Parameters & Min. ACF & Max. ACF & MC Error & Post. Mean & Post. Std. Error \\
\hline$\alpha_{1}$ & -0.12 & 0.35 & 33.93 & 1249.93 & 479.89 \\
$\alpha_{2}$ & -0.04 & 0.22 & 4.31 & 183.73 & 60.94 \\
$\alpha_{3}$ & -0.06 & 0.21 & 2.58 & 147.12 & 36.55 \\
$\tau$ & -0.06 & 0.18 & 1.71 & 57.43 & 24.23 \\
$\eta$ & -0.07 & 0.15 & 0.00 & 0.85 & 0.07 \\
$\sigma_{\epsilon}^{2}$ & -0.09 & 0.38 & 7015.32 & 182712.30 & 99211.54 \\
$\sigma_{w}^{2}$ & -0.12 & 0.25 & 59646.44 & 888470.00 & 843528.00 \\
$\sigma_{\delta}^{2}$ & -0.15 & 0.30 & 0.00 & 0.06 & 0.00 \\
$\rho$ & 0.13 & 0.95 & 0.00 & 0.93 & 0.03 \\
$R^{*}$ (\# distinct components) & -0.12 & 0.09 & 0.09 & 6.05 & 1.27 \\
$\beta_{1}$ (averages) & -0.12 & 0.26 & 77.11 & 63.56 & 1090.47 \\
$\beta_{2}$ (averages) & -0.13 & 0.13 & 46.52 & 41.19 & 657.92 \\
$\beta_{3}$ (averages) & -0.14 & 0.13 & 47.63 & 72.89 & 673.54 \\
$\gamma_{11}$ (averages) & -0.13 & 0.14 & 0.04 & -0.03 & 0.60 \\
$\gamma_{12}$ (averages) & -0.12 & 0.16 & 0.07 & -0.10 & 0.99 \\
$\gamma_{13}$ (averages) & -0.13 & 0.13 & 0.06 & -0.20 & 0.89 \\
$\gamma_{21}$ (averages) & -0.12 & 0.19 & 0.03 & -0.02 & 0.38 \\
$\gamma_{22}$ (averages) & -0.14 & 0.14 & 0.07 & -0.14 & 0.96 \\
$\gamma_{23}$ (averages) & -0.14 & 0.13 & 0.04 & -0.03 & 0.61 \\
$\gamma_{31}$ (averages) & -0.13 & 0.16 & 0.03 & -0.04 & 0.39 \\
$\gamma_{32}$ (averages) & -0.13 & 0.13 & 0.04 & -0.05 & 0.57 \\
$\gamma_{33}$ (averages) & -0.14 & 0.13 & 0.04 & -0.06 & 0.57 \\
\hline
\end{tabular}

$\gamma_{i j}(t) ; t=1, \ldots, T$, and $i, j=1,2,3$, we found that only 16 out of 2,565 null hypotheses were rejected after controlling for the expected false discovery rate (FDR) of Benjamini and Hochberg (1995) at $q=0.05$. This provides support for the notion that our MCMC samples were predominantly indeed from the target posterior distributions.

S-3.2.2. Restricted sub-model $\mathcal{M}_{D P}^{(1)}$. We report here details on the convergence assessment of the generated MCMC samples using the (best performing) sub-model $\mathcal{M}_{D P}^{(1)}$. Once again, we note that informal tools such as simple trace plots and autocorrelation plots showed no evidence of non-convergence of the Markov Chains to the stationary distributions. Table S-11 summarizes the estimated ACFs and the Monte Carlo standard errors. Once again, there is not much evidence of lack of convergence of the MCMC samples. Similar to the case with $\mathcal{M}_{D P}$ we examined the posterior samples of the $\gamma_{i j}(t)$ s more closely and calculated the $p$-values of the K-S test statistics: these are displayed in Figure S-6. There is a preponderance of cases where the $p$-values are substantially away from zero. 


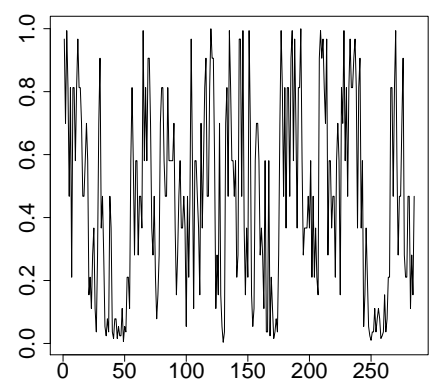

(a) $\gamma_{11}(t)$

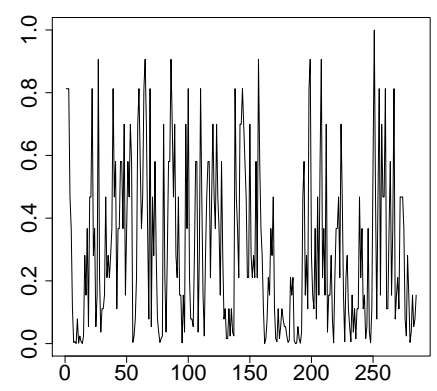

(d) $\gamma_{21}(t)$

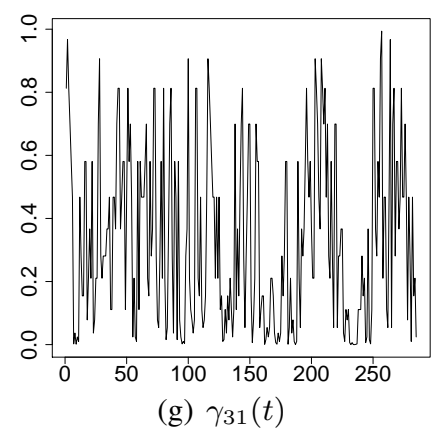

(g) $\gamma_{31}(t)$

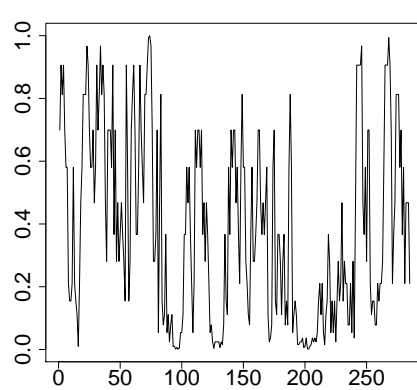

(b) $\gamma_{12}(t)$

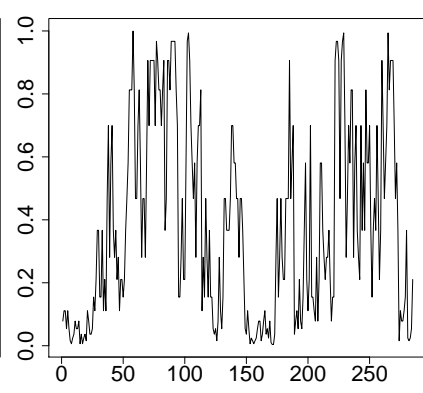

(e) $\gamma_{22}(t)$

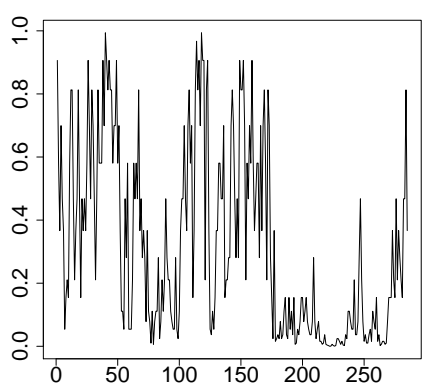

(c) $\gamma_{13}(t)$

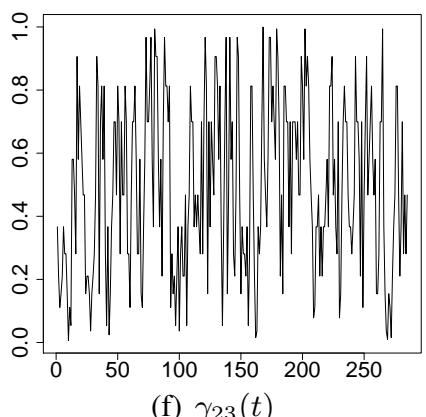

(f) $\gamma_{23}(t)$

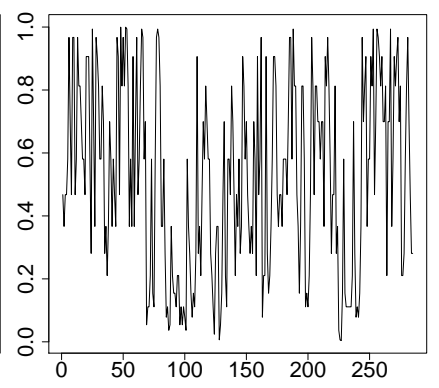

(h) $\gamma_{32}(t)$

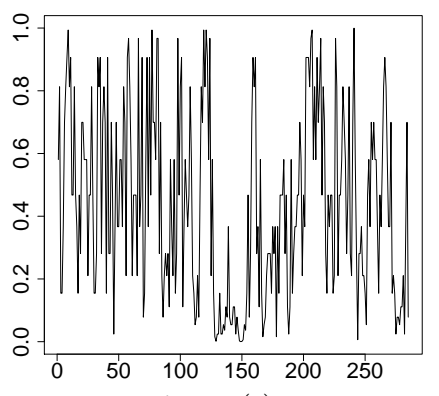

(i) $\gamma_{33}(t)$

FIG S-5. Real fMRI data analysis: p-values of Kolmogorov-Smirnov test for stationarity of the $M C M C$ samples associated with the posterior distributions of $\gamma_{i j}(t) s$ using $\mathcal{M}_{D P}$.

Indeed a quantitative assessment revealed that only 54 out of 2,280 null hypotheses were rejected after controlling for FDR at $q=0.05$, reassuring that most of our MCMC samples are indeed from the target posterior densities.

S-3.3. Analysis using $\mathcal{M}_{A R}$. Figure S-7 displays the estimated marginal posterior densities of the regional influences over time, obtained using $\mathcal{M}_{A R}$. Note that there is a clear oscillatory trend in $\gamma_{11}(t)$, but this trend is missing from most 
TABLE S-11

Stroop task data analysis: Convergence details of the unknown variables associated with $\mathcal{M}_{D P}^{(1)}$ (the best model).

\begin{tabular}{|c|ccccc|}
\hline Parameters & Min. ACF & Max. ACF & MC Error & Post. Mean & Post. Std. Error \\
\hline$\alpha_{1}$ & -0.11 & 0.43 & 30.19 & 1149.65 & 426.96 \\
$\alpha_{2}$ & -0.14 & 0.20 & 4.58 & 206.40 & 64.82 \\
$\alpha_{3}$ & -0.22 & 0.19 & 2.36 & 157.64 & 33.41 \\
$\tau$ & -0.14 & 0.15 & 1.60 & 46.41 & 22.69 \\
$\eta$ & -0.12 & 0.14 & 0.00 & 0.84 & 0.08 \\
$\sigma_{\epsilon}^{2}$ & -0.12 & 0.47 & 5851.48 & 157665.50 & 82752.36 \\
$\sigma_{w}^{2}$ & -0.12 & 0.22 & 30495.73 & 688151.60 & 431274.70 \\
$\sigma_{\delta}^{2}$ & -0.10 & 0.37 & 0.00 & 0.06 & 0.01 \\
$\rho$ & 0.19 & 0.94 & 0.00 & 0.91 & 0.05 \\
$R^{*}$ (\# distinct components) & -0.11 & 0.22 & 0.09 & 4.70 & 1.21 \\
$\beta_{1}$ (averages) & -0.12 & 0.29 & 69.37 & 64.10 & 981.02 \\
$\beta_{2}$ (averages) & -0.13 & 0.14 & 42.80 & 35.58 & 605.22 \\
$\beta_{3}$ (averages) & -0.14 & 0.14 & 42.72 & 57.75 & 604.20 \\
$\gamma_{11}$ (averages) & -0.13 & 0.16 & 0.04 & -0.04 & 0.53 \\
$\gamma_{12}$ (averages) & -0.15 & 0.18 & 0.06 & -0.11 & 0.83 \\
$\gamma_{13}$ (averages) & -0.15 & 0.15 & 0.05 & -0.14 & 0.75 \\
$\gamma_{21}$ (averages) & -0.14 & 0.25 & 0.03 & -0.02 & 0.39 \\
$\gamma_{22}$ (averages) & -0.13 & 0.17 & 0.06 & -0.09 & 0.83 \\
$\gamma_{23}$ (averages) & -0.13 & 0.13 & 0.04 & -0.04 & 0.58 \\
$\gamma_{31}$ (averages) & -0.13 & 0.24 & 0.03 & -0.05 & 0.38 \\
$\gamma_{32}$ (averages) & -0.13 & 0.13 & 0.04 & -0.02 & 0.55 \\
\hline
\end{tabular}

of the other $\gamma_{i j}(t)$ s. There is some evidence of periodic temporal effects in $\gamma_{31}(t)$ and $\gamma_{33}(t)$, but very little for the others, notably $\gamma_{12}(t), \gamma_{13}(t), \gamma_{22}(t), \gamma_{23}(t)$ and $\gamma_{32}(t)$. Note also that the $\gamma_{i j}(t)$ s have support that vary very widely.

S-3.4. Analysis using additional models. We provide here details on performance evaluations when using additional sub-models of $\mathcal{M}_{D P}$ as mentioned in Section 4.1.1, as well as using $\mathcal{M}_{A R}$ and $\mathcal{M}_{R W}$ and the restricted version found by Bhattacharya, Ho and Purkayastha (2006) to be the best model. These models are:

1. $\mathcal{M}_{D P}^{(4)}: \gamma_{33}(t)=\gamma_{21}(t)=0 \forall t$.

2. $\mathcal{M}_{D P}^{(5)}: \gamma_{33}(t)=\gamma_{31}(t)=0 \forall t$.

3. $\mathcal{M}_{D P}^{(6)}: \gamma_{33}(t)=\gamma_{31}(t)=\gamma_{21}(t)=0 \forall t$.

4. $\mathcal{M}_{D P}^{(7)}: \gamma_{23}(t)=0 \forall t$.

5. $\mathcal{M}_{D P}^{(8)}: \gamma_{31}(t)=\gamma_{32}(t)=0 \forall t$.

6. $\mathcal{M}_{R W}$ : unrestricted random walk model.

7. $\mathcal{M}_{R W}^{-}: \mathcal{M}_{R W}$ with $\gamma_{31}(t)=\gamma_{32}(t)=0 \forall t$. 


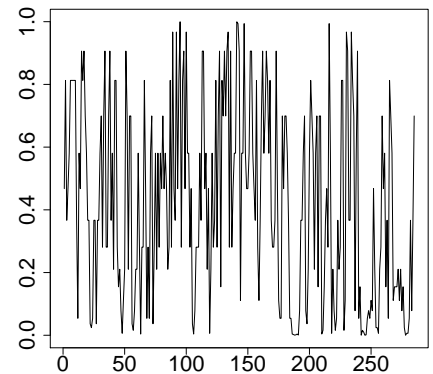

(a) $\gamma_{11}(t)$

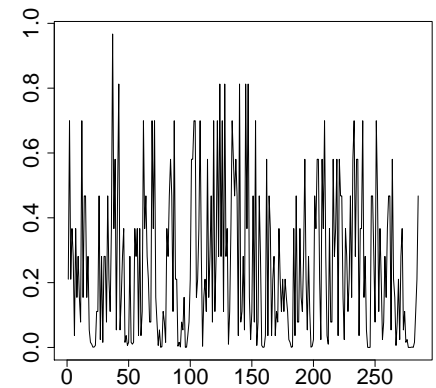

(d) $\gamma_{21}(t)$

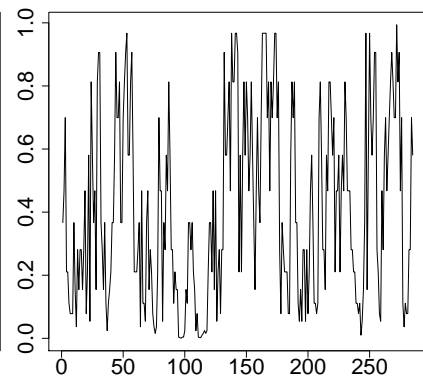

(b) $\gamma_{12}(t)$

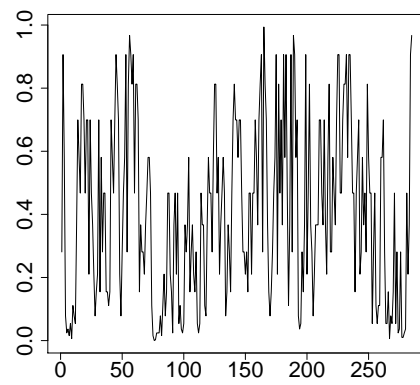

(e) $\gamma_{22}(t)$

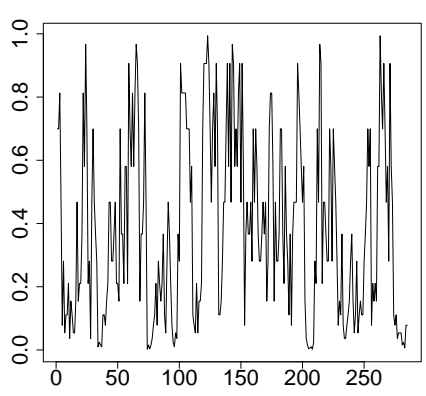

(c) $\gamma_{13}(t)$

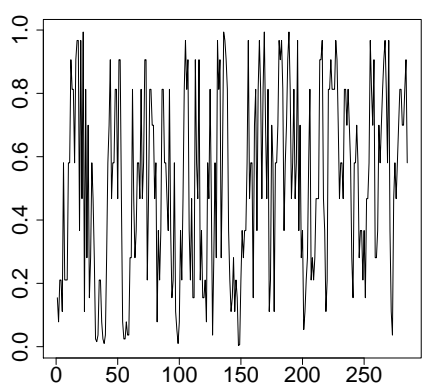

(f) $\gamma_{23}(t)$

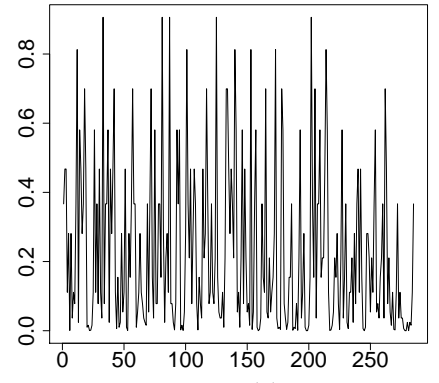

(g) $\gamma_{31}(t)$

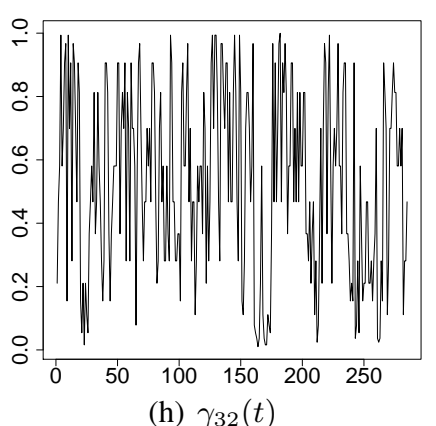

(h) $\gamma_{32}(t)$

FIG S-6. Real fMRI data analysis: $p$-values of the $K$-S tests of stationarity of the MCMC samples associated with the posterior distributions of $\gamma_{i j}(t) s$ using $\mathcal{M}_{D P}^{(1)}$.

8. $\mathcal{M}_{A R}^{-}: \mathcal{M}_{A R}$ with $\gamma_{31}(t)=\gamma_{32}(t)=0 \forall t$.

Tables S-12 and S-13 provide a summary of the evaluated predictive performance of each of these models in terms of the proportions of observed $y$ s included in the 95\% HPD predictive credible intervals and the mean lengths of these intervals. As reported in Section 4.1.1, $\mathcal{M}_{D P}^{(1)}$ is the best model.

S-3.5. Smoothing the modeled BOLD signal. Figure S-8 is a plot of the smoothed modeled BOLD signal obtained upon fitting $x(t)$ with the model $x(t)=A \cos (2 \pi \omega t+$ 


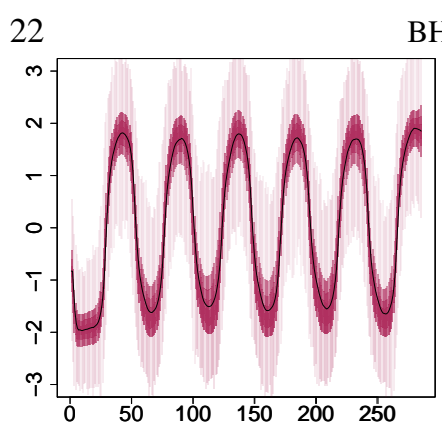

(a) $\gamma_{11}(t)$

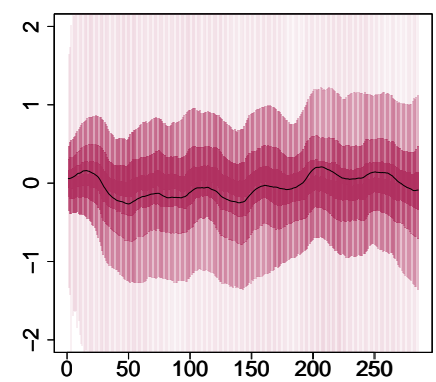

(d) $\gamma_{21}(t)$

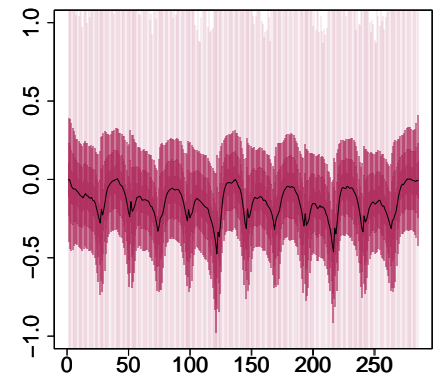

(g) $\gamma_{31}(t)$

BHATTACHARYA AND MAITRA

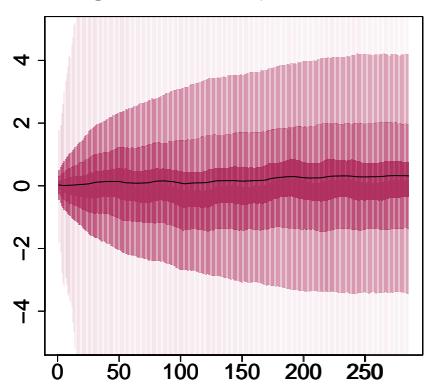

(b) $\gamma_{12}(t)$

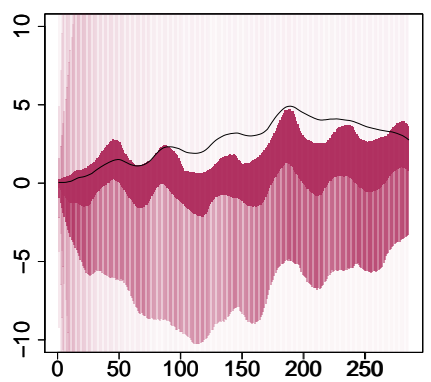

(e) $\gamma_{22}(t)$

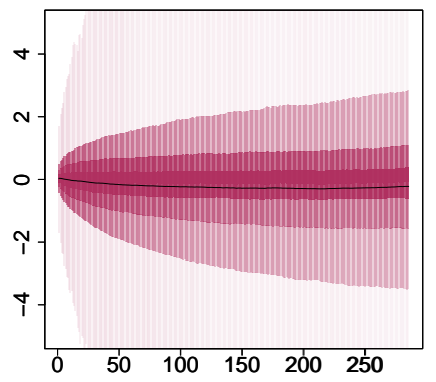

(h) $\gamma_{32}(t)$

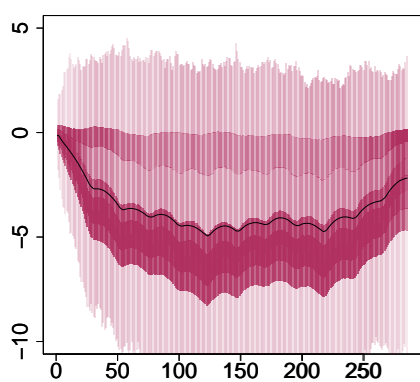

(c) $\gamma_{13}(t)$

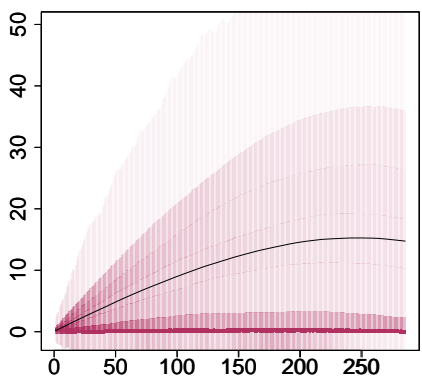

(f) $\gamma_{23}(t)$

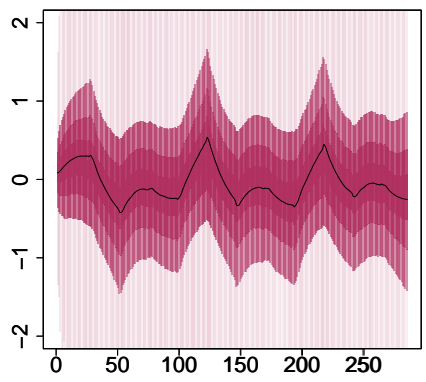

(i) $\gamma_{33}(t)$

FIG S-7. Estimated posterior densities (means in solid lines) of the regional influences over time using $\mathcal{M}_{A R}$.

$\phi)+\psi_{t}$ where $\psi_{t} \stackrel{i i d}{\sim} N\left(0, \sigma_{\psi}^{2}\right), A$ is the amplitude of the time series, and $\omega$ is the oscillation frequency and $\phi$ is a phase shift. The estimated parameter values are $\hat{\omega}=0.02 \hat{A}=0.80$ and $\hat{\phi}=1.16$. Clearly the fitted smoothed signal $\hat{x}(t)$ closely approximates the original time series $x(t)$.

\section{References.}

BARTLETT, M. (1957). A comment on D. V. Lindley's statistical paradox. Bometrika 44533534.

BAYARri, M. J. and Berger, J. O. (1999). Bayesian Statistics 6 Quantifying surprise in the data and model verication 5382. Oxford University Press. 
TABLE S-12

Proportions of observed data included in the $95 \%$ credible intervals of the corresponding posterior predictive distributions upon fitting $\mathcal{M}_{D P}^{(4)}-\mathcal{M}_{D P}^{(8)}, \mathcal{M}_{R W}, \mathcal{M}_{R W}^{-}, \mathcal{M}_{A R}^{-}$to the Stroop task data.

\begin{tabular}{|c|c|c|c|c|c|c|c|c|}
\hline \multirow[t]{2}{*}{$y$} & \multicolumn{8}{|c|}{ Proportion } \\
\hline & $\mathcal{M}_{D P}^{(4)}$ & $\mathcal{M}_{D P}^{(5)}$ & $\mathcal{M}_{D P}^{(6)}$ & $\mathcal{M}_{D P}^{(7)}$ & $\mathcal{M}_{D P}^{(8)}$ & $\mathcal{M}_{R W}$ & $\mathcal{M}_{R W}^{-}$ & $\mathcal{M}_{A R}^{-}$ \\
\hline$y_{1}$ & 0.99 & 0.99 & 1.00 & 1.00 & 0.99 & 1.00 & 0.92 & 0.92 \\
\hline$y_{2}$ & 1.00 & 1.00 & 1.00 & 1.00 & 1.00 & 1.00 & 1.00 & 1.00 \\
\hline$y_{3}$ & 1.00 & 1.00 & 1.00 & 1.00 & 1.00 & 0.93 & 1.00 & 1.00 \\
\hline
\end{tabular}

TABLE S-13

Mean lengths of the $95 \%$ credible intervals of the corresponding posterior predictive distributions upon fitting $\mathcal{M}_{D P}^{(4)}-\mathcal{M}_{D P}^{(8)}, \mathcal{M}_{R W}, \mathcal{M}_{R W}^{-}, \mathcal{M}_{A R}^{-}$to the Stroop task data.

\begin{tabular}{|c||cccccccc|}
\hline$y$ & \multicolumn{7}{|c|}{ Mean Length } \\
& $\mathcal{M}_{D P}^{(4)}$ & $\mathcal{M}_{D P}^{(5)}$ & $\mathcal{M}_{D P}^{(6)}$ & $\mathcal{M}_{D P}^{(7)}$ & $\mathcal{M}_{D P}^{(8)}$ & $\mathcal{M}_{R W}$ & $\mathcal{M}_{R W}^{-}$ & $\mathcal{M}_{A R}^{-}$ \\
\hline$y_{1}$ & $2,689.9$ & $3,503.4$ & $5,370.6$ & $2,737.6$ & $3,856.8$ & $3,217.3$ & $5,842.4$ & $3,749.1$ \\
$y_{2}$ & $2,429.6$ & $3,162.0$ & $4,205.2$ & $2,487.0$ & $3,406.0$ & $3,076.6$ & $4,376.9$ & $3,607.8$ \\
$y_{3}$ & $2,535.5$ & $3,170.0$ & $4,489.5$ & $2,541.9$ & $3,485.9$ & $4,726.9$ & $4,861.4$ & $3,553.5$ \\
\hline
\end{tabular}

BAyARri, M. J. and Berger, J. O. (2000). $p$-values for Composite Null Models (with discussion). Journal of the American Statistical Association 9511271142.

Benjamini, Y. and Hochberg, Y. (1995). Controlling the false discovery rate: a practical and powerful approach to multiple testing. Journal of the Royal Statistical Society 57 289-300.

Bhattacharya, S., Ho, M. R. and Purkayastha, S. (2006). A Bayesian approach to modeling dynamic effective connectivity with fMRI data. NeuroImage 30 794-812.

BROOK, D. (1964). On the distinction between the conditional probability and the joint probability approaches in the specification of nearest-neighbour systems. Biometrika 51 481-483.

CARlin, B. P. and Louis, T. A. (1996). Bayes and empirical bayes methods for data analysis. Chapman and Hall Second Edition.

GEISSER, S. and EDDY, W. F. (1979). A predictive approach to model selection. Journal of the American Statistical Association 74 153-160.

Gelfand, A. E. (1996). Model determination using sampling-based methods. In Markov Chain Monte Carlo in Practice (W. GILKS, S. RichARDSON and D. SPIEGELHALTER, eds.). Interdisciplinary Statistics 145-162. Chapman and Hall, London.

Gelfand, A. E. and DEY, D. K. (1994). Bayesian model choice: Asymptotics and exact calculations. Journal of the Royal Statistical Society B 56 501-514.

Gelman, A., Meng, X. L. and Stern, H. S. (1996). Posterior predictive assessment of model fitness via realized discrepancies (with discussion). Statistica Sinica 6733807.

Guttman, I. (1967). The use of the concept of a future observation in goodness-of-fit problems. Journal of the Royal Statistical Society. Series B 29 83-100.

KASS, R. E. and RAFTERY, R. E. (1995). Bayes Factors. Journal of the American Statistical Association 90 773-795.

Meng, X. L. (1994). Posterior predictive $p$-values. Annals of Statistics 22 1142-1160.

Peruggia, M. (1997). On the variability of case-deletion importance sampling weights in the Bayesian linear model. Journal of the American Statistical Association 92 199-207.

Robert, C. P. and Casella, G. (2004). Monte Carlo Statistical Methods. Springer-Verlag, New 


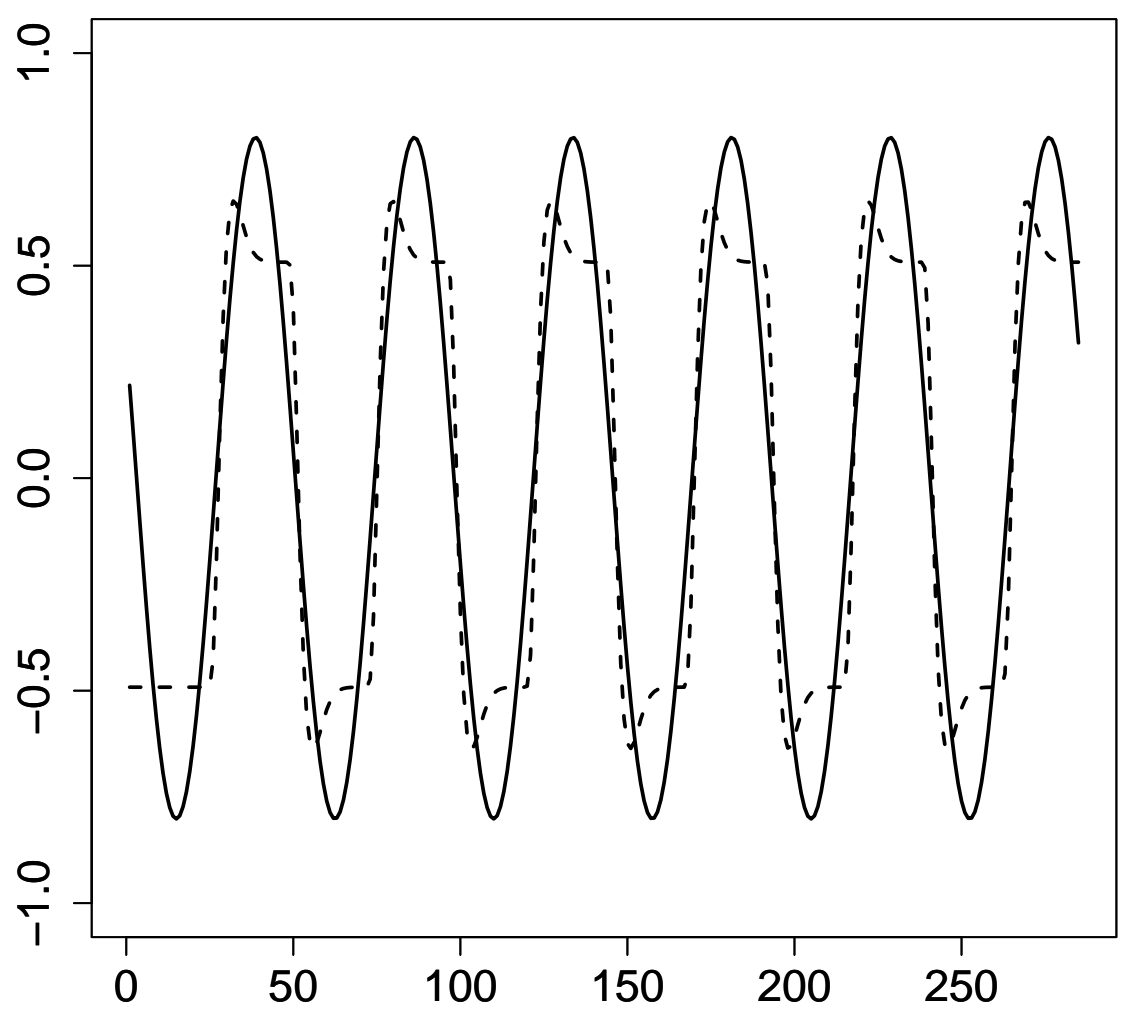

FIG S-8. Plot of the fitted smoothed modeled BOLD $\hat{x}(t)$ (solid line) along with its original $x(t)$ (broken line), both plotted against time.

York.

RUBIN, D. B. (1984). Bayesianly justifiable and relevant frequency calculations for the applied statistician. Annals of Statistics 12 1151-1172.

BAYESIAN AND INTERDISCIPLINARY RESEARCH UNIT DEPARTMENT OF STATISTICS AND STATISTICAL LABORATORY INDIAN STATISTICAL INSTITUTE 203, B. T. ROAD, KOLKaTA 700108 IOWA STATE UNIVERSITY

E-MAIL: sourabh@isical.ac.in

AMES, IA 50011-1210

E-MAIL: maitra@iastate.edu 